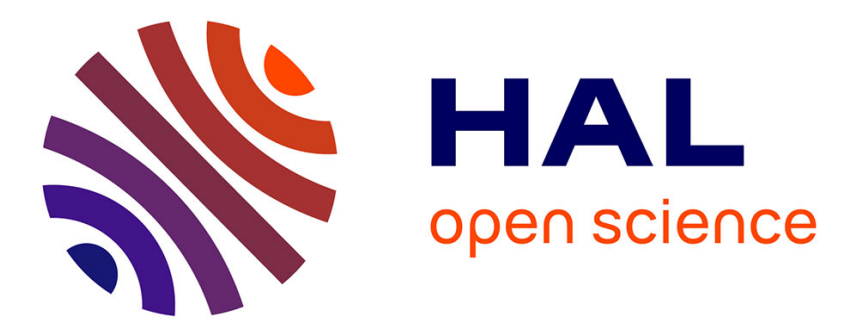

\title{
New developments of the Gas Research Institute method for the permeability measurement of porous media
}

\author{
Yves Jannot, Alain Degiovanni, Christian Moyne, Didier Lasseux
}

\section{To cite this version:}

Yves Jannot, Alain Degiovanni, Christian Moyne, Didier Lasseux. New developments of the Gas Research Institute method for the permeability measurement of porous media. Review of Scientific Instruments, 2021, 92 (6), pp.065102. 10.1063/5.0043915 . hal-03248974

\section{HAL Id: hal-03248974 https://hal.science/hal-03248974}

Submitted on 16 Jun 2021

HAL is a multi-disciplinary open access archive for the deposit and dissemination of scientific research documents, whether they are published or not. The documents may come from teaching and research institutions in France or abroad, or from public or private research centers.
L'archive ouverte pluridisciplinaire HAL, est destinée au dépôt et à la diffusion de documents scientifiques de niveau recherche, publiés ou non, émanant des établissements d'enseignement et de recherche français ou étrangers, des laboratoires publics ou privés. 


\title{
New developments of the Gas Research Institute method for the permeability measurement of porous media
}

Rev. Sci. Instrum. 92, 065102 (2021); doi: 10.1063/5.0043915

Yves Jannot, ${ }^{2}$ Alain Degiovanni, ${ }^{1,2}$ Christian Moyne, ${ }^{1}$ and Didier Lasseux ${ }^{3, a)}$

\author{
AFFILIATIONS \\ ${ }^{1}$ Université de Lorraine, CNRS, LEMTA, 54000 Nancy, France \\ ${ }^{2}$ Université Internationale de Rabat, Pôle Energie, LERMA, Rocade Rabat-Salé, Sala Al Jadida 11100, Morocco \\ ${ }_{3}^{3}$ I2M, UMR 5295, CNRS, University Bordeaux, 351, Cours de la Libération, 33405 CEDEX Talence, France
}

a) Author to whom correspondence should be addressed: didier.lasseux@u-bordeaux.fr. Tel.: +33540 003421

\begin{abstract}
This work reports on new developments of an unsteady-state method to measure the permeability and porosity of permeable porous materials, extending the so-called Gas Research Institute method beyond its classical use. These extensions allow one to carry out measurements on samples having a cylindrical shape in the case of one- or three-dimensional (or axisymmetric) flows. They rely on the derivation of the quasianalytical solutions that are required to interpret the experimental data by solving an inverse problem. The only simplifying assumption is that the compressibility of the probing gas can be treated as a constant over the range of pressure variations during measurement. The relevance of the method, together with the validity of this hypothesis, is checked over a wide range of porosity and permeability values through a sensitivity analysis and a comparison with direct numerical simulations of the complete initial boundary value problem. The efficiency of the method is further illustrated with experiments performed on four different porous materials in one-dimension and axisymmetric configurations for permeabilities ranging between $10^{-14}$ and $10^{-19} \mathrm{~m}^{2}$. The potential capability of diagnosing heterogeneous and/or anisotropic materials is highlighted. These new developments open the way for further extensions to samples of arbitrary shapes or partially saturated by a trapped fluid.
\end{abstract}

\section{INTRODUCTION}

Permeability and porosity are fundamental properties of natural or manufactured porous materials, which are of interest in numerous applications in the domains of petroleum, civil, and chemical engineering to cite only a few. These quantities are of central importance for oil or gas recovery predictions, efficiency assessment of cap rocks or concrete for confinement sites, or air renewal design for bio-based thermal insulation materials in buildings, for instance. While the porosity, denoted by $\varepsilon$ in what follows, is a static quantity that characterizes the pore volume fraction of the material, the permeability is a signature of its resistance to viscous fluid flow. Its value can range over several orders of magnitude, typically from $\sim 10^{-12}$ to $\sim 10^{-20} \mathrm{~m}^{2}$ for hydrocarbon-bearing reservoir rocks or from $\sim 10^{-17}$ to $10^{-20} \mathrm{~m}^{2}$ for concrete.
For a homogeneous isotropic material, the permeability can be considered as a scalar quantity (denoted by $k$ in the following) that can be measured via several different methods depending on its value (see Refs. 1 and 2 and references therein).

For large to moderate values of $k$ (down to roughly $10^{-18} \mathrm{~m}^{2}$ ), a steady-state method can be employed, ${ }^{3,4}$ consisting in performing a $1 \mathrm{D}$ stationary flow in a sample whose end faces are submitted to a pressure difference, while the sides are sealed. Measurements of the pressure drop and flow rate across the sample allow the determination of $k$ on the basis of Darcy's law. However, as $k$ becomes smaller, the method requires instruments of increasing precision in order to accurately measure smaller flow rates. ${ }^{5}$ In addition, the time required to reach steady-state (gas) flow increases as $1 / k$. For these reasons, unsteady-state methods may be preferred for $k$ typically smaller than $\sim 10^{-18} \mathrm{~m}^{2}$. Among them, the pulse-decay, 
initially proposed by Brace et al. ${ }^{6}$ and further developed over the two following decades, ${ }^{5-9}$ is one of the most popular ones. For this method, a 1D cylindrical sample is employed. It is sealed on its outer peripheral surface, and each extremity is connected to a reservoir. Starting from a pressure equilibrium state in the gas saturating the system, a pressure pulse is imposed in the upstream reservoir, and the pressure difference between the upstream and downstream tanks is registered over time. The permeability is estimated from this pressure signal, usually in the long-time range, using a simplified model in which the dynamic viscosity, density, and compressibility of the flowing gas are considered as constant. ${ }^{6}$ Another method, referred to as the oscillating pore pressure method, was devised in the $1990 \mathrm{~s}^{10,11}$ (see also Ref. 12). In this method, a sinusoidal pressure is imposed in the upstream reservoir, and the steady-state periodic pressure signal is recorded in the downstream tank. Estimation of the permeability relies on the upstream/downstream pressure amplitude ratio along with their phase shift.

More recently, an alternative method, the step-decay, was proposed. ${ }^{13,14}$ The experimental device for this method is the same as for the pulse-decay, but several successive pressure pulses are imposed in the upstream reservoir. The method does not require a state back to equilibrium between each pulse (which may be long) as would be needed if several different pressure levels would be used in a pulse-decay experiment. The step-decay is a potential-potential type of method. It is based on the transfer function estimate between the upstream and downstream pressures yielding the physical parameters of the sample, which are free of errors due to uncertainties on the upstream parameters of the experiment, such as dead volumes and thermal effects resulting from sudden pressure variations.

The gas slippage effect often occurs when measurements are performed at certain pore pressure levels and permeabilities. ${ }^{15,16}$ In that case, $k$ is an apparent permeability, which includes the Klinkenberg effect, ${ }^{17}$ and is given by $k=k_{l}\left(1+\frac{b}{p}\right)$, where $k_{l}$ is the intrinsic permeability, $b$ is the Klinkenberg coefficient, and $P$ is the average pressure over the sample at which $k$ is estimated. When steady-state experiments are used, the identification of $k_{l}$ and $b$ is achieved by repeating the experiment at several average pressures. Conversely, the step-decay was shown to allow the simultaneous identification of $k_{l}, b$, and $\varepsilon$ from a single experiment.

In all the methods mentioned above, the measurement is performed on a cylindrical sample that must be perfectly sealed on its sides. This is commonly achieved by inserting the sample in a soft impermeable jacket over which a confining pressure is applied in order to avoid a preferential flow-path, which would seriously bias the sample permeability estimate (in particular, in the range of small $k$ ). However, most of the time, the stress induced by the confining pressure induces a deformation resulting in a permeability modification.

A technique, which advantageously circumvents this last difficulty, was developed in the early 1990s by the Gas Research Institute (GRI). ${ }^{18,19}$ The eponymous technique, also referred to as the pressure fall-off method or inflow measurement method, consists in placing the porous sample to be characterized in a closed tank. A pressure increment is imposed in the tank, and the ensuing pressure decrease due to the gas penetration in the porous material is registered from which the permeability and porosity are extracted. This method has been applied to crushed porous materials of various forms and dimensions ${ }^{20,21}$ or to cylindrical samples. ${ }^{22}$ In the former case, the porous grains are assimilated to spheres to simplify the interpretation with semi-analytical solutions. ${ }^{23}$ In the latter case, analytical solutions were derived in two configurations: (i) for samples featuring a ratio of the diameter to the length much smaller than unity for which the pressure within the sample is uniform along the sample axis and only depends on the radial position; (ii) for samples sealed on their peripheral surface for which the pressure is the only function of the axial position. Approximated models were also proposed, applicable at early and late times of the pressure signal. In the latter case, which is the most widely used, a normalized pressure decline curve is derived as a function of time, and the permeability is estimated from the slope at late times. The porosity is not identified with this method. In all the models, an instantaneous initial pressure excitation (under the form of a Dirac delta) is assumed. Moreover, the gas compressibility, $\beta=\frac{1}{P}$, is taken as constant over the pressure decay period. This hypothesis is also retained in this work after testing its validity.

A limitation toward a more extensive use of the GRI method certainly lies in the absence of operational interpretative models available to date, the models being limited to the two situations mentioned above. The objective of the present work is hence to propose new developments of this method by deriving a complete two-dimensional model for a cylindrical sample, regardless of its aspect ratio, as well as complete one- and three-dimensional models for parallelepipedic samples, using integral transforms on the initial boundary value problem for the gas pressure evolution during the measurement. These models allow accounting for the pressure variation in the sample over the entire experimental duration in order to extract the parameters of interest. A detailed analysis of the reduced sensitivities of the pressure signal to the parameter $D=\frac{k}{\varepsilon}$ and to the porosity is carried out, showing that these two parameters can be simultaneously extracted from the time evolution of the pressure variation in the tank (i.e., at the sample surface). This is further illustrated by performing a parameter estimation on synthetic pressure signals obtained from a direct numerical simulation of the model for which the compressibility is not constant, assuming, in addition, that the apparent permeability obeys a Klinkenberg relationship. These tests show that both parameters, $D$ and $\varepsilon$, are correctly estimated (with a better accuracy for the former) and that the constant compressibility hypothesis does not induce any significant bias in the estimation. The analysis also indicates that the extended GRI method, together with the estimation procedure developed here, allows the measurement of permeabilities in the interval $10^{-14}-10^{-19}$ $\mathrm{m}^{2}$ as proved by measurements carried out on four different porous materials with permeabilities in this range.

With these purposes in mind, this article is organized as follows: In Sec. II, the principle of the method is recalled. The $1 \mathrm{D}$ and $3 \mathrm{D}$ models for a parallelepipedic sample as well as the $2 \mathrm{D}$ axisymmetric one for a cylindrical sample of circular cross section are developed. Section III is dedicated to the parameter estimation procedure using an inverse method. Section IV is devoted to experiments. After presenting the experimental device and protocol, measurements performed on samples of two different insulating materials and two different rocks are reported. Conclusions are drawn in Sec. V. 


\section{PRINCIPLE OF THE METHOD AND MODELS}

Before developing the models for parallelepipedic and cylindrical samples of circular cross section, which are used in the remainder of this work, it is of interest to recall the main features of the GRI method.

\section{A. Principle}

A schematic diagram of the device employed in a GRI method, similar to that envisaged by Profice et al. ${ }^{23}$ or Cui et al. ${ }^{5}$ to carry out measurements on crushed porous media, is represented in Fig. 1.

Two temperature regulated tanks (tank 0 and tank 1 of respective volume $V_{0}$ and $V_{1}$ ) are connected through a tubing equipped with a valve $V_{1}$, which is closed at the initial stage of the experiment. The sample, in which permeability, $k$, and porosity, $\varepsilon$, are to be measured, is placed in tank 1 . The pressure of the gas present in tank 0 is set to a different value, $P_{0}$, (larger or smaller) than that of the same gas present in tank 1 . At $t=0$, the valve $V_{1}$ is opened and then closed (at time $t=t_{c}$ ) in order to put both tanks in communication over a short period of time. This creates a pressure pulse in tank 1 , whose evolution over time, $P_{1}(t)$, back to equilibrium due to the presence of the porous sample, is recorded. As shown in what follows, the shape of the pressure evolution curve allows one to determine the parameter $D=\frac{k}{\varepsilon}$ of the material, regardless of the amplitude of the pressure variation. In addition, the porosity, $\varepsilon$, can also be identified independently from $D$.

In the following, the method to extract these parameters is detailed considering two types of sample shapes, namely, parallelepipedic and cylindrical of circular cross section. While the former can be of interest for anisotropic materials, if the sample edges are parallel to the direction of anisotropy, the latter is of interest for isotropic (and poorly permeable) materials.

\section{B. 1D and 3D Cartesian models}

\section{1. $1 D$ case}

In order to ensure a $1 \mathrm{D}$ gas transfer within the material, all faces of the sample of length $L$ are sealed, except the one orthogonal to the direction for which permeability is to be estimated, as schematically represented in Fig. 2. The direction of measurement $(x)$ is supposed to be a principal direction of the permeability tensor.

Assuming that the pressure inside the sample, denoted $P_{i}$, is uniform, equal to that in tank 1 at the initial stage, and that the gas obeys ideal gas law, the initial boundary value problem (IBVP) for $P \equiv P(x, t)$ is given by ${ }^{23}$

$$
\begin{gathered}
\frac{\partial P}{\partial t}-\frac{1}{\varepsilon \mu} \frac{\partial}{\partial x}\left(k P \frac{\partial P}{\partial x}\right)=0, \\
\text { B.C.1 } \quad P=P_{1}(t) \text { at } x=0, \\
\text { B.C.2 } \quad \frac{\partial P}{\partial x}=0 \text { at } x=L, \\
\text { I.C. } \quad P=P_{i} \text { at } t=0 .
\end{gathered}
$$

In Eq. (1), $\mu$ represents the gas dynamic viscosity, whereas the apparent permeability, $k$, in the direction of measurement may include a Klinkenberg correction, i.e.,

$$
k=k_{l}\left(1+\frac{b}{P}\right)
$$

with $k_{l}$ being the intrinsic permeability and $b$ being the Klinkenberg coefficient (in Pa). To arrive at Eq. (1), the mass and the steady form of Darcy's law were combined (see Refs. 24 and 25 for the details).

Boundary condition B.C. 2 in Eq. (3) expresses the fact that the gas flux is zero at $x=L$ since this face is sealed.

The pressure evolution in the system is initiated by opening the valve $V_{1}$ allowing a gas flux, $\dot{q}_{m 0}(t)$, in (or out of) tank 1 . The flux is maximum at the valve opening and may be taken as zero at $t=t_{c}$ when the pressure, denoted $P_{\max }$, tends to equilibrate between the two tanks. It is hence reasonable to represent $\dot{q}_{m 0}(t)$ by the triangle function,

$$
\dot{q}_{m 0}(t)=\frac{2 q_{0}}{t_{c}}\left(1-\frac{t}{t_{c}}\right)\left[1-\gamma\left(t-t_{c}\right)\right], \quad t \leq t_{c},
$$

Pressure $P_{0}$

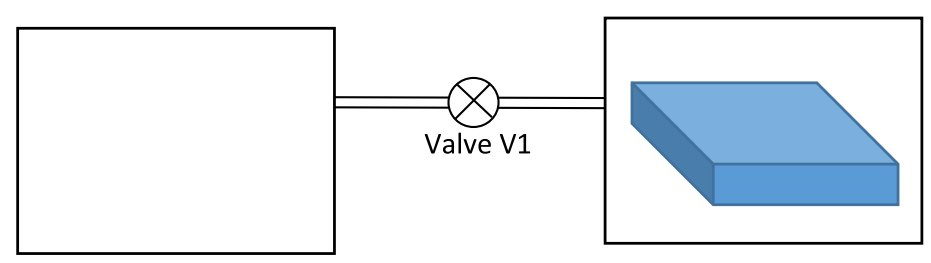

Tank 0 (volume $V_{0}$ )

\section{Pressure $P_{1}$}

Tank 1 (volume $V_{1}$ ) enclosing the sample (volume $V$, permeability $k$, porosity $\varepsilon$ )

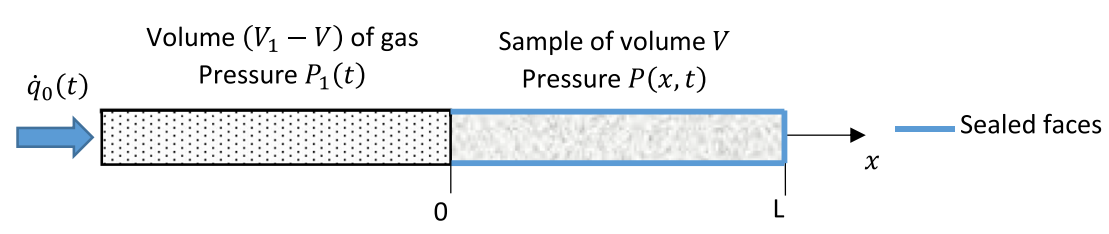

FIG. 1. Schematic representation of the experimental device employed in a GRI method.
FIG. 2. 1D configuration for a parallelepipedic sample. 
where $\gamma$ is the Heaviside function and $q_{0}$ is the mass of gas entering or leaving tank 1 over $t_{c}$.

In order to close the formulation of the IBVP, it is necessary to make explicit the coupling between the pressures $P_{1}(t)$ in tank 1 and $P$ inside the sample (see B.C. 1) resulting from the excitation. This is achieved by expressing the gas mass flux balance at $t$ in the system including tank 1 and the sample, which gives

$$
\frac{M}{R T}\left(V_{1}-V\right) \frac{d\left(P_{1}-P_{i}\right)}{d t}=-\varepsilon V \frac{M}{R T} \frac{d\left(P_{m}-P_{i}\right)}{d t}+\dot{q}_{m 0} .
$$

Here, $P_{m}$ represents the average pressure inside the sample at $t$, i.e.,

$$
P_{m}=\frac{1}{L} \int_{0}^{L} P(x, t) d x
$$

In Eq. (7), $M$ is the gas molar mass and $R=8.314 \mathrm{~J} \mathrm{~mol}^{-1} \mathrm{~K}^{-1}$ is the ideal gas constant.

The pressure equation (1) is non-linear and, under this form, does not admit any analytical solution. To make further progress, it is of interest to assume that $P$ experiences small variations compared to its average value over the measurement period, denoted $P_{c}$, and is given by

$$
P_{c}=\frac{1}{t_{f}-t_{c}} \int_{t_{c}}^{t_{f}} P_{m}(t) d t,
$$

with $t_{f}$ being the time at which the experiment ends. This assumption is consistent with the fact that the gas compressibility, $\beta$, can be considered as constant, taken as $\beta=\frac{1}{P_{c}}$. Under these circumstances, it is reasonable to use the approximation $k P \cong k_{l}\left(1+\frac{b}{P_{c}}\right) P_{c}$ in Eq. (1), which can be simplified to the following form:

$$
\frac{\partial^{2} P}{\partial x^{2}}=\frac{1}{a} \frac{\partial P}{\partial t} .
$$

Here, $a$ denotes the diffusivity (in $\mathrm{m}^{2} \mathrm{~s}^{-1}$ ) given by

$$
a=\frac{k}{\varepsilon \mu \beta}=\frac{D}{\mu \beta}=\frac{P_{c}}{\mu} D .
$$

The solution of the above IBVP can then be carried out in the Laplace domain. Denoting $\theta(x, p)=\mathcal{L}[\bar{P}(x, t)]=\int_{0}^{\infty} \bar{P} \exp (-p t) d t$ as the Laplace transform of $\bar{P}=P-P_{i}$, the solution of Eq. (1) is given by $^{26}$

$$
\theta(x, p)=A \cosh (q x)+B \sinh (q x),
$$

with $q=\sqrt{\frac{p}{a}}$. The Laplace transform of B.C. 1 in Eq. (2) yields

$$
\theta_{1}=\theta(0, p)=\mathcal{L}\left[\bar{P}_{1}(t)\right]=A .
$$

Moreover, B.C. 2 [Eq. (3)] gives

$$
B=-A \tanh (q L) .
$$

The Laplace transform of the mass flux balance expressed in Eq. (7) yields

$$
\Phi_{1}=\frac{M\left(V_{1}-V\right)}{R T} p \theta(0, p)+\varepsilon V \frac{M}{R T} p \theta_{m}
$$

where $\Phi_{1}=\mathcal{L}\left[\dot{q}_{m 0}\right]$ and $\theta_{m}=\mathcal{L}\left[P_{m}\right]$. By making use of Eqs. (8) and (12), $\theta_{m}$ is given by

$$
\begin{aligned}
\theta_{m} & =\frac{1}{L} \int_{0}^{L} \theta(x, p) d x=\frac{1}{L} \int_{0}^{L}[A \cosh (q x)+B \sinh (q x)] d x \\
& =\frac{A \tanh (q L)}{q L} .
\end{aligned}
$$

When this result is introduced back into Eq. (15), the expression of $A$ can be obtained as

$$
A=\Phi_{1} \frac{R T}{M} \frac{1}{\left(V_{1}-V\right) p+\varepsilon \sqrt{p a} \frac{V}{L} \tanh (q L)}=\theta_{1} .
$$

For the subsequent use of this solution, it is convenient to write it as

$$
\theta_{1}=\frac{X_{1}}{p} \frac{1}{1+\frac{X_{2}}{X_{3} \sqrt{p}} \tanh \left(X_{3} \sqrt{p}\right)},
$$

in which

$$
\begin{gathered}
X_{1}=\Phi_{1}(p) \frac{R T}{M\left(V_{1}-V\right)}, \\
X_{2}=\frac{\varepsilon V}{V_{1}-V}, \\
X_{3}=L \sqrt{\frac{1}{a}} .
\end{gathered}
$$

If $\dot{q}_{m 0}(t)$ is taken as the triangle function expressed in Eq. (6), $\Phi_{1}$ is given $b^{27}$

$$
\Phi_{1}(p)=\frac{2 q_{0}}{t_{c}}\left[\frac{1}{p}-\frac{1-\exp \left(-p t_{c}\right)}{t_{c} p^{2}}\right] .
$$

From Eq. (18), it can be observed that $\theta_{1}$ behaves as $\frac{X_{1}}{p}$ when $p$ tends to infinity. It can hence be deduced that $X_{1}$ represents the extremum, $P_{\max }$, of the pressure $P_{1}$ imposed by the initial pulse.

Finally, the pressure in Tank $1, P_{1}(t)$, is obtained from an inverse Laplace transform of Eq. (18) making use, for instance, of the De Hoog algorithm. ${ }^{28}$ The solution depends on the three parameters $X_{1}, X_{2}$, and $X_{3}$ and, more precisely, on $\varepsilon$ (involved in $X_{2}$ ) and $D$ (through $X_{3}$ ).

It must be noted that the development in the $1 \mathrm{D}$ case presented here was carried out for a sample of length $L$, assuming that only one end is left open. Nevertheless, the solution is still applicable when both opposite faces of the sample are left open. However, $V$ and $L$ in the solution given in Eqs. (18)-(21) must, respectively, be understood as half the sample volume and length in that case. Leaving one or the two faces open to gas transfer may be of interest depending on the sample length and permeability in order to design a long enough experiment allowing an accurate parameter estimate.

\section{3D case}

If the material can be considered as isotropic and homogeneous, a 3D experiment, for which all the six faces are left open to gas transfer, can be of interest. The methodology to derive the solution, albeit more cumbersome, is similar to the one detailed above 
in the $1 \mathrm{D}$ case. The steps to arrive at the solution are provided in Appendix A. If the dimensions of the sample are $\ell, L$, and $h$, the solution, $\theta_{1}$, on $P_{1}-P_{i}$ in the Laplace domain takes the following form:

$$
\theta_{1}=\frac{\mathrm{X}_{1}}{p} \frac{1}{1+64 X_{2} \sum_{n=1}^{\infty} \sum_{m=1}^{\infty} F_{n m}} .
$$

In this expression, $X_{1}$ and $X_{2}$ are given by Eqs. (19) and (20), while $F_{n m}$ has the following expression:

$$
\begin{aligned}
F_{n m}= & \left(\frac{1}{\alpha_{n}^{2} L \ell}+\frac{1}{\gamma_{m}^{2} L \ell}\right) \frac{1}{\delta_{n m}^{2} L \ell}\left\{\left[\frac{\delta_{n m}{ }^{2} h^{2}}{\alpha_{n}^{2} h^{2}+\gamma_{m}^{2} h^{2}}-1\right]\right. \\
& \left.\times \frac{2}{\delta_{n m} h} \frac{\sinh \left(\delta_{n m} \frac{h}{2}\right)}{\cosh \left(\delta_{n m} \frac{h}{2}\right)}+1\right\},
\end{aligned}
$$

with

$$
\begin{gathered}
\alpha_{n}=\frac{(2 n-1) \pi}{\ell}, \\
\gamma_{m}=\frac{(2 m-1) \pi}{L}, \\
\delta_{n m}=\sqrt{\alpha_{n}^{2}+\gamma_{m}^{2}+\frac{p}{a}},
\end{gathered}
$$

and $a$ given in Eq. (11). Again, $P_{1}(t)$ is obtained from an inverse Laplace transform of Eq. (23). Apart from the dimensions of the sample, this solution depends on the physical parameters $\varepsilon$ and $D$ (respectively, involved in $X_{2}$ and $\delta_{n m}$ ), as in the $1 \mathrm{D}$ case.

\section{2D axisymmetric model}

In this configuration schematically represented in Fig. 3, a cylindrical sample, of length $L$ and of circular cross section of radius $r_{1}$, is placed in tank 1 with all the faces left open. As for the $3 \mathrm{D}$ case envisaged above, this configuration is of interest when the material is homogeneous and isotropic. For convenience, the inner radius and height of the tank are denoted by $r_{c}$ and $L_{c}$, respectively.

With the origin of coordinates placed at the center of the sample and assuming again that the variations of $P$ during the experiment $\left(t_{c} \leq t \leq t_{f}\right)$ remain small compared to $P_{c}$ [see Eq. (9)], the IBVP is given by the following system of equations:

$$
\frac{\partial^{2} P}{\partial x^{2}}+\frac{1}{r} \frac{\partial P}{\partial r}+\frac{\partial^{2} P}{\partial r^{2}}=\frac{1}{a} \frac{\partial P}{\partial t}
$$

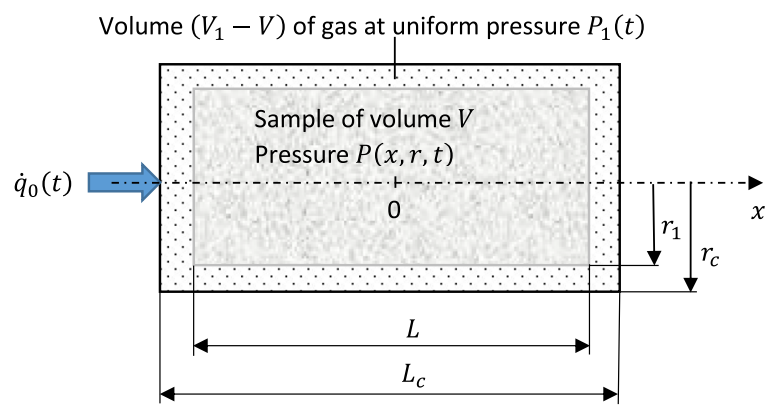

FIG. 3. Schematic representation of the axisymmetric configuration.
B.C.1

$$
\frac{\partial P}{\partial x}=0 \text { at } x=0
$$

B.C. 2

$$
P=P_{1}(t) \text { at } x=\frac{L}{2} \text { and } r=r_{1},
$$

I.C.

$$
P=P_{i} \text { at } t=0,
$$

with $a$ being the diffusivity defined in Eq. (11). A condition must be added here, imposing that $P$ remains finite at $r=0$. The mass balance of the gas in the system, including the sample and the tank, leads to Eq. (7), in which $P_{m}$ represents the mean pressure in the sample at $t$ [see Eq. (8)] and $\dot{q}_{m 0}$ is the gas flux between tank 0 and tank 1 at the initial stage of the experiment $\left(0 \leq t \leq t_{c}\right)$.

The solution to this problem is obtained using a double integral (Laplace and Fourier) transform. Details on the derivation are provided in Appendix B, yielding

$$
\theta_{1}(p)=\frac{X_{1}}{p} \frac{1}{1+8 X_{2} \sum_{n=1}^{\infty} \frac{1}{\delta_{n}{ }^{2} L^{2}}\left[2 \frac{L}{r_{1}}\left(\frac{\delta_{n} L}{\alpha_{n}{ }^{2} L^{2}}-\frac{1}{\delta_{n} L}\right) \frac{I_{1}\left(\delta_{n} r_{1}\right)}{I_{0}\left(\delta_{n} r_{1}\right)}+1\right]} .
$$

In this expression, $I_{0}$ and $I_{1}$ are the zeroth and first order Bessel functions of the first kind, $\theta_{1}$ denotes the Laplace transform of $P_{1}-P_{i}, X_{1}$ and $X_{2}$ are again given in Eqs. (19) and (20), while $\alpha_{n}$ and $\delta_{n}$ have the following expressions:

$$
\alpha_{n}=(2 n-1) \frac{\pi}{L}
$$

$$
\delta_{n}=\sqrt{\alpha_{n}^{2}+\frac{p}{a}}
$$

where $a$ is the diffusivity expressed in Eq. (11). The solution for $P_{1}(t)$ is finally obtained from an inverse Laplace transform of Eq. (32). It depends again on $\varepsilon$ and $D$ (contained in $X_{2}$ and $\delta_{n}$ ) as for the parallelepipedic sample in $1 \mathrm{D}$ and 3D.

At this point of the analysis, a difficulty regarding the value of $\beta=\frac{1}{P_{c}}$, with $P_{c}$ being given in Eq. (9), must be addressed. Indeed, with this definition, $P_{c}$ depends on $P$, which means that a solution for $P$ can only be obtained in an iterative manner. For the sake of simplicity, in practice, this may be simplified by making use of an approximated constant value of $P_{c}$ based on the observation that $P_{1}$ varies first from $P_{i}$ to $P_{\max }$, while the valve $V_{1}$ remains open and then would reach the value $P_{f}$ at final equilibrium. This leads to propose the following approximation for $P_{c}$ :

$$
P_{c}=\frac{\frac{P_{i}+P_{\max }}{2}+P_{f}}{2} .
$$

Note that $P_{f}$ does not necessarily corresponds to $P_{1}\left(t_{f}\right)$. The validity of this approximation will be assessed in Sec. III A 3. It should be noted that $P_{\max }$ is not known a priori and is difficult to measure in a real experiment. As a consequence, the idea is to estimate $q_{0}$ involved in $X_{1}$ [see Eq. (19)] in addition to $D=\frac{k}{\varepsilon}$ present in $X_{3}, \delta_{n m}$, or $\delta_{n}$ [Eqs. (21) and (27) or Eq. (34)] and $\varepsilon$ involved in $X_{2}$ [Eq. (20)] from the time evolution record of $P_{1}(t)$. 


\section{PARAMETER ESTIMATION}

The purpose is now to investigate how the parameters $q_{0}, \varepsilon$, and $\frac{k}{\varepsilon}$, on which the solutions always depend, can be estimated from a recording of $P_{1}(t)$ using the quasi-analytical solutions presented in Sec. II. The feasibility of this estimation may be checked by analyzing, first, the signal amplitude and, second, its reduced sensitivities to the parameters to be estimated. The numerical tests reported in this section were carried out using the following parameters: $V=2 \cdot 10^{-3} L, V_{1}=1.2 \mathrm{~V}, T=298 \mathrm{~K}$, and $P_{i}=10^{5} \mathrm{~Pa}$. Moreover, air was assumed as the probing gas for which $M=29 \mathrm{~g} \mathrm{~mol}^{-1}$ and $\mu=1.8 \cdot 10^{-5} \mathrm{~Pa}$. The values of $q_{0}$ and $t_{c}$ were chosen such that $P_{\max } \cong 1.3 \cdot 10^{5} \mathrm{~Pa}$ using the triangle function given in Eq. (6) with $t_{c}=0.2 \mathrm{~s}$.

\section{A. The 1D flow case in a parallelepipedic sample}

A thorough analysis is carried out in the case of a parallelepipedic sample in which flow only takes place along the sample axis, as described in Sec. II B 1.

\section{Range of measurement}

The solution of Eq. (18), once inverse Laplace transformed, was computed for four values of $L$, namely, $L=5,10,50$, and $100 \mathrm{~mm}$; a porosity $\varepsilon=0.1$, yielding $P_{c}=1.175 \cdot 10^{5} \mathrm{~Pa}$; a permeability, $k$, ranging between $10^{-15}$ and $10^{-21} \mathrm{~m}^{2}$; and $t_{f}=3600 \mathrm{~s}$. This time limit was chosen as a reasonable period over which temperature can be easily regulated in a real experiment without any sophisticated device.

The corresponding signals of $\overline{P_{1}}(t)=P_{1}(t)-P_{i}$ are reported in Fig. 4. If a criterion for a significant signal amplitude, $P_{\max }-P_{f}$, is
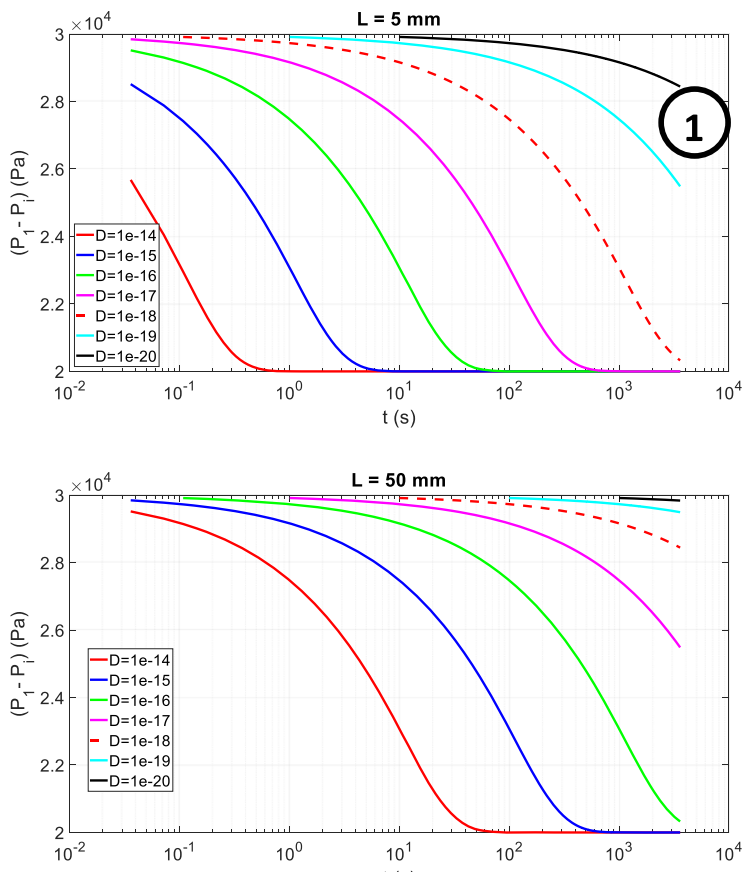

$t(s)$ thought to be $5 \%$ of $P_{\max }-P_{i}$, which seems reasonable for a standard pressure transducer, this figure allows one to conclude that for the measurement period and the parameters under consideration, the estimation of $D$ can be envisaged in the range from $D=10^{-20}$ $\mathrm{m}^{2}$ (with $L=5 \mathrm{~mm}$ ) to $D=10^{-14} \mathrm{~m}^{2}$ (with $L=100 \mathrm{~mm}$ ) (see curves, respectively, labeled 1 and 2 in Fig. 4). Nevertheless, to further evaluate the estimation feasibility, it is necessary to carry out a sensitivity analysis.

An example of the pressure evolution inside a sample with $L$ $=100 \mathrm{~mm}, k=10^{-14} \mathrm{~m}^{2}$, and $\varepsilon=0.9$ is provided in Fig. 5 . In the first half of the sample $(0 \leq x \leq 50 \mathrm{~mm})$, the pressure evolution is nonmonotonic as it starts to increase and then decreases to reach the final equilibrium value $P_{f}$, whereas in the second half $(50 \leq x \leq 100$ $\mathrm{mm}$ ), the pressure increases from $P_{i}$ to $P_{f}$ [see Fig. 5(a)]. Moreover, as shown in Fig. 5(b) where the pressure evolution is represented inside the tank (at $x=0$ ) at $x=10$ and $x=20 \mathrm{~mm}$ at the early stage of the experiment, i.e., for $0 \leq t \leq 1 \mathrm{~s}$, a pressure change is noticeable close to the sample entrance (at $x=10 \mathrm{~mm}$ ). Even if this observation corresponds to a permeability in the upper range of those envisaged here, it justifies why $\dot{q}_{m 0}$ should be carefully taken into account in the derivation of the model to interpret the experimental data.

\section{Analysis of the reduced sensitivities}

The reduced sensitivity of $P_{1}(t)$ to a parameter $X_{i}$ is defined by $X_{i} \frac{\partial P_{1}}{\partial X_{i}}$. Estimation of the parameter $X_{i}$ is possible if the reduced sensitivity to $X_{i}$ is larger than the sensibility of the pressure transducer employed to record $P_{1}(t)$ and if it is not proportional to the reduced sensitivity of any other parameter $X_{j}, i \neq j$, to be estimated. The
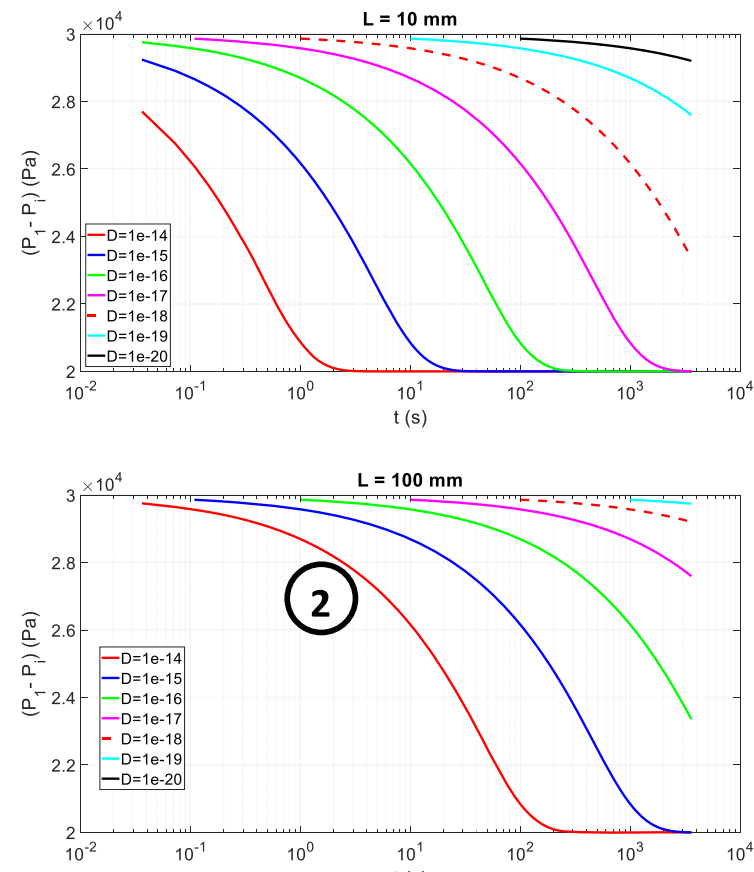

$\mathrm{t}(\mathrm{s})$

FIG. 4. 1D simulations of $\bar{P}_{1}(t)$ for four values of the sample length $L=5,10,50$, and $100 \mathrm{~mm}$ and $D=\frac{k}{\varepsilon}$ ranging from $10^{-14}$ to $10^{-20} \mathrm{~m}^{2} . \varepsilon=0.1$. 
a)

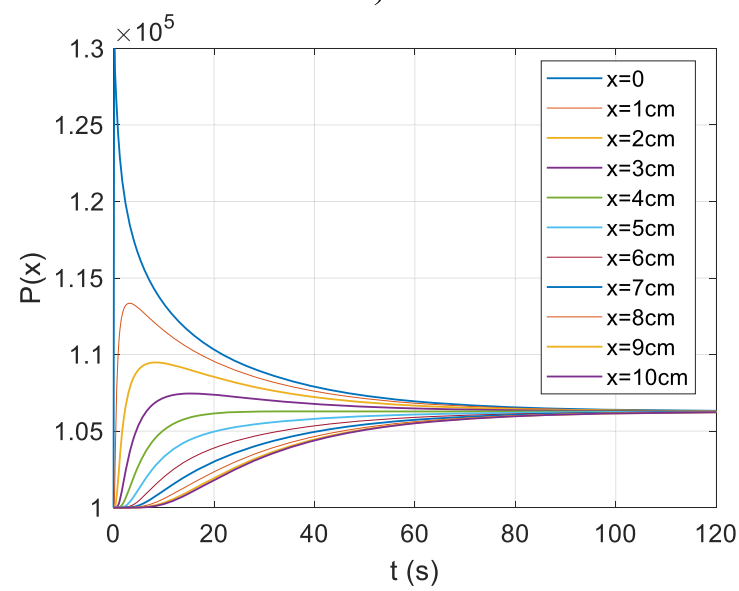

b)

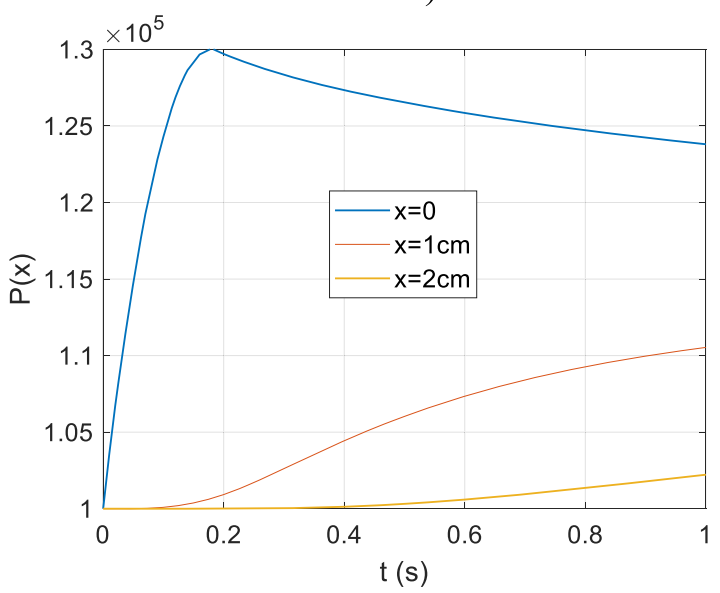

FIG. 5. Pressure evolution in the $1 \mathrm{D}$ case within a sample with $k=10^{-14} \mathrm{~m}^{2}, \varepsilon=0.9$, and $L=100 \mathrm{~mm}$ (a) in the range $0 \leq t \leq 120 \mathrm{~s}$ every $10 \mathrm{~mm}$ within the sample and (b) in the tank $(x=0)$ at $x=10$ and $x=20 \mathrm{~mm}$ for $0 \leq t \leq 1 \mathrm{~s}$.

reduced sensitivities of $P_{1}(t)$ to the parameters $q_{0}, \varepsilon$, and $D=\frac{k}{\varepsilon}$ were computed to verify whether they are correlated or not in order to consider their simultaneous estimation from an inverse procedure.

The time evolution of the reduced sensitivities in six different cases is reported in Fig. 6 along with the corresponding pressure signals $\overline{P_{1}}(t)$. This figure shows that, in all cases, the sensitivity to $D$ is not correlated with the sensitivities to $q_{0}$ and $\varepsilon$, which means that $D$ can be estimated from $\overline{P_{1}}(t)$ in the ranges of $k$ and $\varepsilon$ considered here. For a large porosity (i.e., $\varepsilon=0.9$ ) and a large permeability (i.e., $k=10^{-14} \mathrm{~m}^{2}$ ) sensitivities to $q_{0}$ and $\varepsilon$ become correlated after a short period of time. This indicates that the accuracy on $q_{0}$ and $\varepsilon$ would decrease in these limits and that it would be preferable to measure $\varepsilon$ separately, for instance, with a pycnometry experiment as described by Bal et al. ${ }^{29}$ and for which a larger value of $P_{\max }$ can be employed. Nevertheless, these limits are barely reached in practical situations.

\section{Parameter estimation}

In the $1 \mathrm{D}$ case investigated here, the three parameters to be estimated from $P_{1}(t)$ are $q_{0}$ [involved in $X_{1}$, see Eq. (19)], $X_{2}$, and $X_{3}$ [see Eqs. (20) and (21)]. These estimations are performed through a minimization of the quadratic difference between the recorded $\left(P_{1 \exp }\right)$ and simulated $\left(P_{1 \text { mod }}\right)$ values of $P_{1}$ at $t_{c} \leq t_{i} \leq t_{f}, i=1, \ldots, N$, defined as $\sum_{n=1}^{N}\left[P_{1 \exp }\left(t_{i}\right)-P_{1 \bmod }\left(t_{i}\right)\right]^{2}$. This is performed using a Levenberg-Marquart algorithm. ${ }^{30}$ The values of $D=\frac{k}{\varepsilon}$ and $\varepsilon$ are then, respectively, obtained from

$$
\begin{gathered}
D=\frac{\mu \beta L^{2}}{X_{3}{ }^{2}}, \\
\varepsilon=X_{2} \frac{V_{1}-V}{V} .
\end{gathered}
$$

Equation (36) shows that $D$ does not explicitly depend on $V_{1}, V, t_{c}$, $q_{0}$, and $T$ and hence that these parameters do not need to be known accurately in the inverse procedure to estimate $D$.
At this point, it is of interest to investigate the validity of the linearization of Eq. (1) yielding Eq. (10) and to the solution in Eq. (18). To do so, the flow problem was solved in its complete form considering the pressure equation (1) instead of its linearized version, which means that the compressibility is not assumed to be constant. The solution of this problem was carried out using Comsol Multiphysics in order to obtain $P_{1}(t)$ as a result of a numerical experiment. Moreover, a Klinkenberg-corrected permeability, as given in Eq. (5), was considered for these experiments, assuming that the Klinkenberg coefficient, $b$, follows a correlation proposed by Jones,

$$
b=0.189 k_{l}^{-0.36} \text {, }
$$

where $b$ is in $\mathrm{Pa}$ and $k_{l}$ in $\mathrm{m}^{2}$.

More specifically, numerical modeling was carried out with a Comsol Multiphysics module allowing to use a pressure dependent permeability. The geometrical configuration was exactly that reported in Fig. 2. Equations (1)-(5) were solved in the sample (volume $V$ ). In addition, the flux equation (6) was enforced by choosing $q_{0}$ such that $P_{\max }=1.3 \cdot 10^{5} \mathrm{~Pa}$ and $t_{c}=0.2 \mathrm{~s}$ and was applied as a boundary condition at the entrance of the $1 \mathrm{D}$ domain. All other parameters were taken to their values recalled at the beginning of Sec. III. The computational domain was meshed using 129 elements, a value that satisfies mesh convergence. The default solver was employed.

Tests were performed in six different cases for which $L, k_{l}$, and $\varepsilon$, along with the corresponding values of $P_{c}$ obtained from Eq. (9), took values reported in Table I.

The numerical results obtained on $P_{1}(t)$ were subsequently used as experimental data from which the estimated values, $\varepsilon_{\text {est }}$ and $k_{\text {est }}$, were determined from the inverse procedure using the solution of the linearized model [Eq. (18)] in which $P_{c}$ in the expressions of $\beta$ and $k$ is given by Eq. (35). The values of $\varepsilon_{e s t}$ and $k_{\text {est }}$ are also reported in Table I, together with those of $k$ computed from Eq. (5) with $P=P_{c}$ and the relative errors on $D, k$, and $\varepsilon$. 

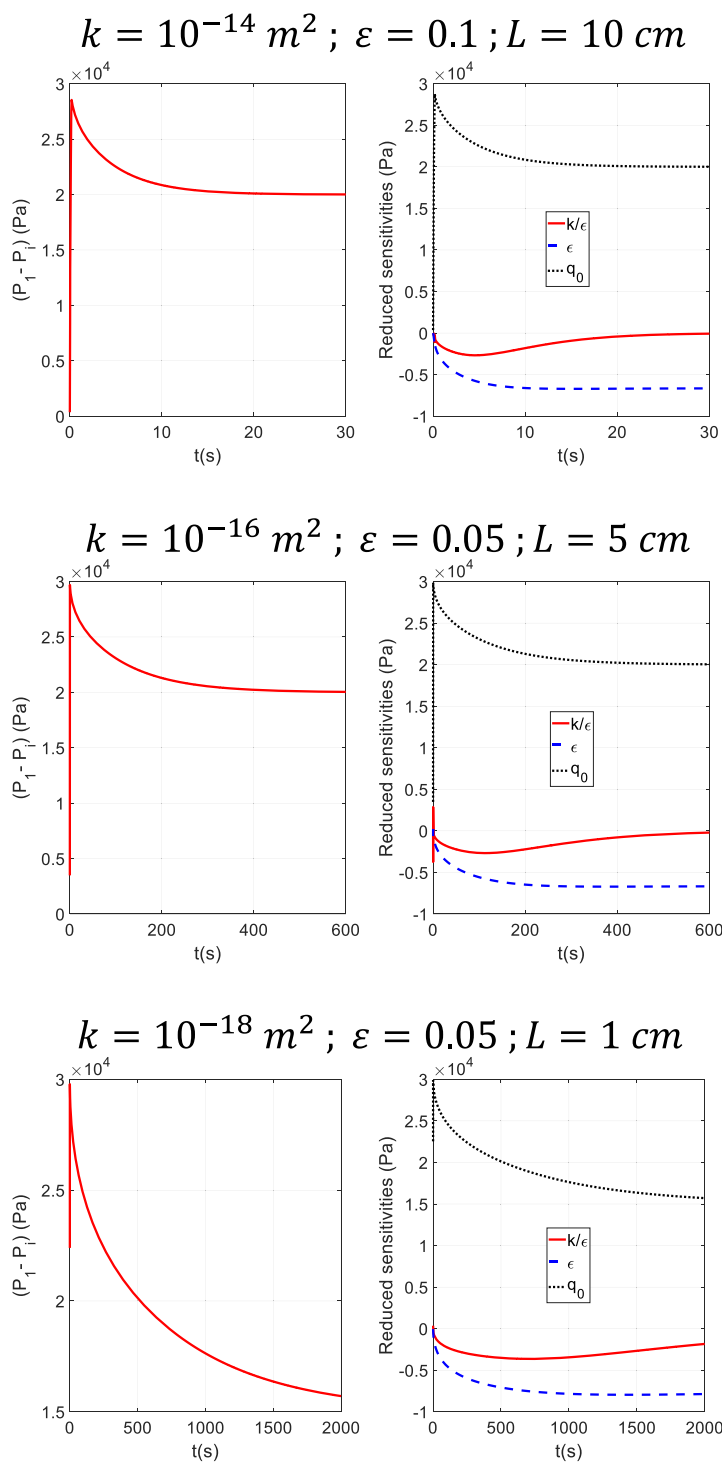
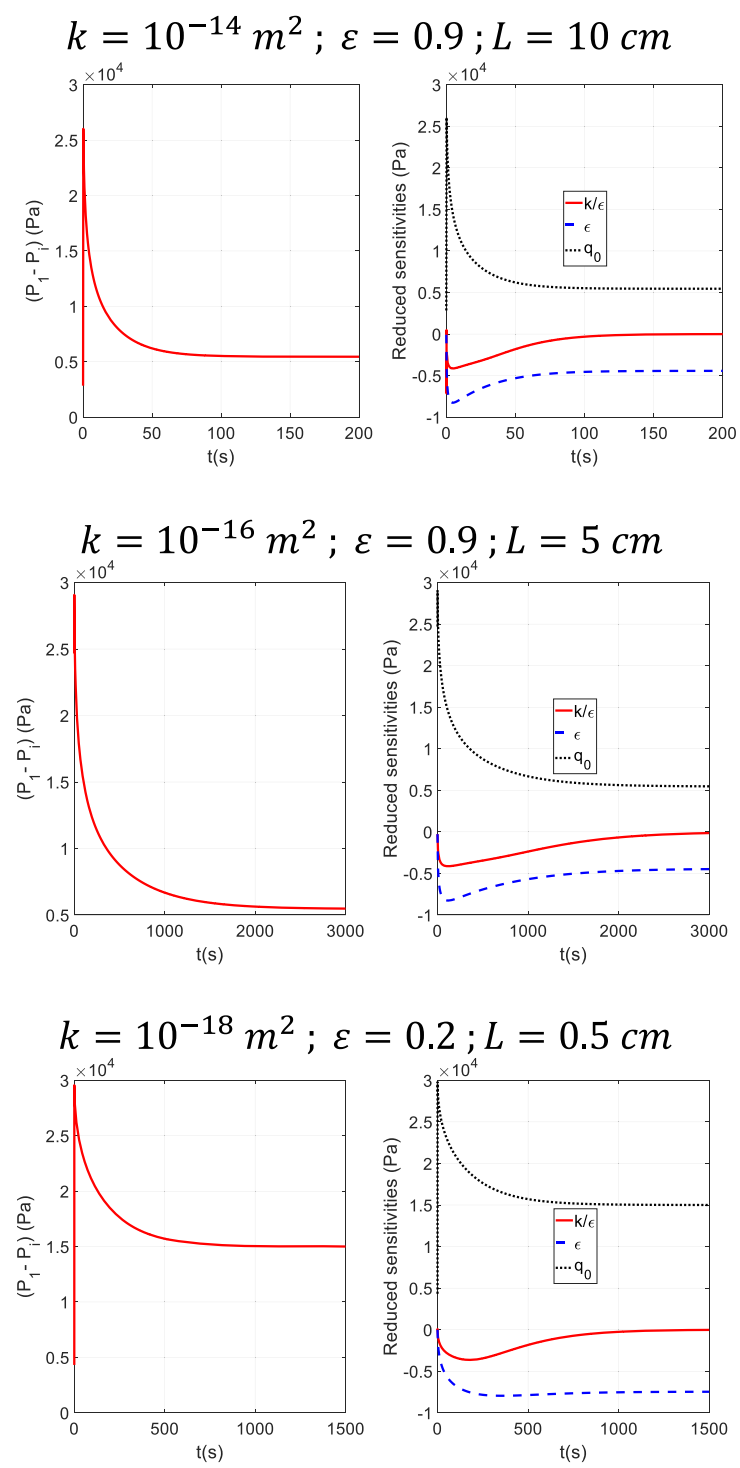

FIG. 6. Evolution of $\bar{P}_{1}(t)$ and of its sensitivities to $q_{0}, \varepsilon$, and $D=\frac{k}{\varepsilon}$ for different values of $\varepsilon, k$, and $L$.

The largest relative error between the estimated and input values of $\varepsilon$ is observed for $\varepsilon=0.9$, as expected from the sensitivity analysis. Correspondingly, the relative error between the estimated and computed values of $k$ is also maximum in this range of porosity. It must be noted, however, that the relative error on $D$, in these cases, is much smaller than that on $k$. This results from the fact that $D_{e s t}$ is the parameter that is directly obtained from the inverse procedure, whereas $k_{\text {est }}$ is computed a posteriori with $\varepsilon_{e s t}$ and hence includes errors from this last parameter. For smaller values of $\varepsilon$, the relative error remains smaller than $0.6 \%$ on $k$ and is barely noticeable on $\varepsilon$. These errors justify the use of the linearized model involving a constant compressibility that corresponds to the pressure $P_{c}$ approximated with Eq. (9) that is also employed to compute $k$ when this parameter is pressure dependent according to a Klinkenberg relationship.

For case 2, the evolution of $P_{1}$, obtained from simulations with Comsol Multiphysics $\left(P_{1 \exp }\right)$ solving the complete problem (i.e., using a permeability that includes Klinkenberg effects and without assuming a constant compressibility), is compared to the prediction $\left(P_{1 \text { mod }}\right)$ from the quasi-analytical solution [Eq. (18)] in which compressibility and permeability are assumed to be constant. This comparison is depicted in Fig. 7 . The residues, $P_{1 \exp }-P_{1 \text { mod }}$, are also reported in this figure showing the excellent agreement between the two up until the final time at which equilibrium is reached. This further illustrates the performance of the linearized model and of the inverse procedure. 
TABLE I. Parameters used for the simulations and results of the estimated values. 1D case.

\begin{tabular}{|c|c|c|c|c|c|c|c|}
\hline Case No. & & 1 & 2 & 3 & 4 & 5 & 6 \\
\hline$k_{l}$ & $\mathrm{~m}^{2}$ & $10^{-14}$ & $10^{-14}$ & $10^{-16}$ & $10^{-16}$ & $10^{-18}$ & $10^{-18}$ \\
\hline B & $\mathrm{Pa}$ & $0.207 \cdot 10^{5}$ & $0.207 \cdot 10^{5}$ & $1.087 \cdot 10^{5}$ & $1.087 \cdot 10^{5}$ & $5.708 \cdot 10^{5}$ & $5.708 \cdot 10^{5}$ \\
\hline $\mathcal{E}$ & $\cdots$ & 0.1 & 0.9 & 0.05 & 0.9 & 0.05 & 0.2 \\
\hline$L$ & $\mathrm{~m}$ & 0.1 & 0.1 & 0.05 & 0.05 & 0.01 & 0.005 \\
\hline$P_{c}[$ Eq. $(35)]$ & $\mathrm{Pa}$ & $1.182 \cdot 10^{5}$ & $1.106 \cdot 10^{5}$ & $1.194 \cdot 10^{5}$ & $1.104 \cdot 10^{5}$ & $1.196 \cdot 10^{5}$ & $1.150 \cdot 10^{5}$ \\
\hline$\varepsilon_{\text {est }}$ & $\cdots$ & 0.1 & 0.862 & 0.0501 & 0.880 & 0.05 & 0.2 \\
\hline$\frac{\varepsilon_{e s t-\varepsilon}}{\varepsilon}$ & $\%$ & 0.0 & -4.1 & 0.0 & -2.2 & 0.0 & 0.0 \\
\hline$k \stackrel{\varepsilon}{\text { Eq. }}$ (5) with $\left.P=P_{c}\right]$ & $\mathrm{m}^{2}$ & $1.175 \cdot 10^{-14}$ & $1.187 \cdot 10^{-14}$ & $1.910 \cdot 10^{-16}$ & $1.985 \cdot 10^{-16}$ & $5.772 \cdot 10^{-18}$ & $5.963 \cdot 10^{-18}$ \\
\hline$k_{\text {est }}$ & $\mathrm{m}^{2}$ & $1.169 \cdot 10^{-14}$ & $1.116 \cdot 10^{-14}$ & $1.909 \cdot 10^{-16}$ & $1.935 \cdot 10^{-16}$ & $5.777 \cdot 10^{-18}$ & $5.945 \cdot 10^{-18}$ \\
\hline$\frac{k_{\text {est }}-k}{k}$ & $\%$ & -0.6 & -6.0 & -0.1 & -2.5 & 0.1 & 0.3 \\
\hline$D\left[\right.$ Eq. (5) with $\left.P=P_{c}\right]$ & $\mathrm{m}^{2}$ & $1.175 \cdot 10^{-13}$ & $1.319 \cdot 10^{-14}$ & $3.822 \cdot 10^{-15}$ & $2.206 \cdot 10^{-16}$ & $1.155 \cdot 10^{-16}$ & $2.982 \cdot 10^{-17}$ \\
\hline$D_{\text {est }}$ & $\mathrm{m}^{2}$ & $1.169 \cdot 10^{-13}$ & $1.319 \cdot 10^{-14}$ & $3.809 \cdot 10^{-15}$ & $2.181 \cdot 10^{-16}$ & $1.154 \cdot 10^{-16}$ & $2.977 \cdot 10^{-17}$ \\
\hline$\frac{D_{\text {est }} D}{D}$ & $\%$ & -0.5 & 0.0 & -0.3 & -1.1 & -0.1 & -0.2 \\
\hline
\end{tabular}

Since a constant value of $k_{\text {est }}$ yields excellent match of the pressure signal generated with a pressure dependent $k$, it can be concluded that the two constants $k_{l}$ and $b$ involved in $k_{\text {est }}$ cannot be estimated simultaneously from a single experiment (a single value of $P_{c}$ ) using the linearized model as there is not a unique pair of $k_{l}$ and $b$ giving the same value of $k_{\text {est }}$. However, several experiments, at different values of $P_{c}$, can be performed in order to obtain the dependence of $k_{\text {est }}$ on $P_{c}$, which, upon using the Klinkenberg form $k_{\text {est }}$ $=k_{l}\left(1+\frac{b}{P_{c}}\right)$, allows one to determine both $k_{l}$ and $b$. This procedure was further employed in the experiments detailed in Sec. IV.

\section{Uncertainty estimate}

In addition to the errors inherent to the inverse procedure employing a linearized simplified model, errors due to uncertainty

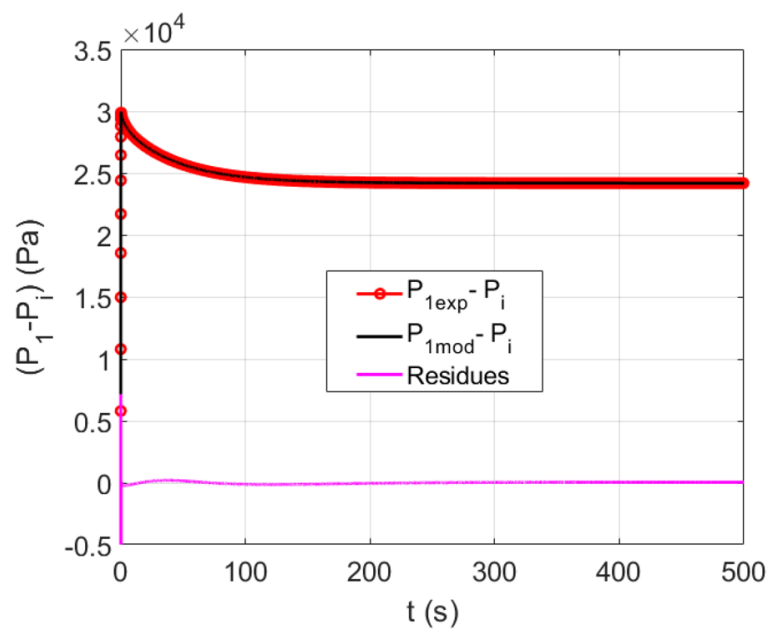

FIG. 7. (Case 2) Pressure evolution in tank 1 obtained from the numerical experiment resulting from the simulation with Comsol Multiphysics $\left(P_{1 \text { exp }}\right)$ and comparison with the solution of the linearized model $\left[P_{1 \text { mod }}\right.$, see Eq. (18)] obtained with the estimated parameters. The residues are denoted as $P_{1 \exp }-P_{1 \text { mod }}$. on the input parameters of the model must also be considered. Equation (36) shows that the relative error, $\Delta D$, induced on $D$ by the errors $\Delta L$ and $\Delta \mu$ on $L$ and $\mu$, respectively, is given by

$$
\frac{\Delta D}{D}=2 \frac{\Delta L}{L}+\frac{\Delta \mu}{\mu} \text {. }
$$

For instance, a relative error of $1 \%$ on $L$ yields a relative error of $2 \%$ on $D$.

Similarly, Eq. (37) shows that the relative error on $\varepsilon$ can be expressed as

$$
\frac{\Delta \varepsilon}{\varepsilon}=\frac{\Delta V}{V}+\frac{\Delta V+\Delta V_{1}}{V_{1}-V} .
$$

The variation of $P_{1}(t)$ is enhanced by a small value of $\left(V_{1}-V\right)$. However, the above relationship indicates that this induces a large error on $\varepsilon$, implying a compromise that is difficult to solve a priori. As an example, if the dimensions of a sample being $0.1 \times 0.1$ $\mathrm{m}^{2}$ in cross section with $L=0.03 \mathrm{~m}$ are known with an absolute error of $0.1 \mathrm{~mm}$, while considering $V_{1}=1.2 \mathrm{~V}$ and a relative error of $1 \%$ on $V$, this leads to $\frac{\Delta V}{V}=\frac{10^{-4}}{0.03}+\frac{10^{-4}}{0.1}+\frac{10^{-4}}{0.1}=0.53 \%$ and $\frac{\Delta \varepsilon}{\varepsilon}$ $=0.0053+\frac{0.0053 \times V+0.01 \times 1.2 \times V}{0.2 \times V}=9.2 \%$. This shows that the expected relative error on $\varepsilon$ induced by uncertainties on the input parameters is larger than that expected on $D$.

With the two estimated parameters being $D$ and $\varepsilon, k$ is computed from $k=D \varepsilon$, which implies

$$
\frac{\Delta k}{k}=\frac{\Delta D}{D}+\frac{\Delta \varepsilon}{\varepsilon} .
$$

In order to decrease the error on $k$, it would be preferable to measure $\varepsilon$ separately with a pycnometry experiment in which only the values of $P_{\max }$ and $P_{f}$ at the final equilibrium are necessary. This can be performed using the same experimental device as in the dynamic experiment under study in this work. ${ }^{29}$ Since there is no constraint on the interpretative model to determine $\varepsilon$ from this experiment, a large pressure variation $P_{\max }-P_{f}$ can be employed to improve the accuracy on $\varepsilon$ and, consequently, on $k$ estimated from the current dynamic procedure. 


\section{B. 2D axisymmetric flow in a cylindrical sample}

The estimation procedure reported above in Sec. III A 3 in the $1 \mathrm{D}$ case was repeated in the case of a cylindrical sample with all faces open to gas flow in order to verify the validity of the approach in that case, making use of the model given in Eq. (32). The series in this solution was approximated with 200 terms to ensure convergence. Numerical experiments were carried out by solving the complete pressure equation (1) (i.e., without assuming a constant compressibility) in the axisymmetric case considering a cylindrical sample of $40 \mathrm{~mm}$ in diameter and $L=60 \mathrm{~mm}$, tank 1 being $44 \mathrm{~mm}$ in diameter and $64 \mathrm{~mm}$ in height. The other physical parameters were those given at the beginning of Sec. III. As in the $1 \mathrm{D}$ case, these simulations were performed using Comsol Multiphysics. Again, the flux equation (6) was enforced by choosing $q_{0}$ such that $P_{\max }=1.3 \cdot 10^{5} \mathrm{~Pa}$ and $t_{c}=0.2 \mathrm{~s}$, and it was applied as a boundary condition at the entrance of the computational domain. A zero flux boundary condition was applied on all other faces of the domain. A triangular mesh, including 4375 elements with a local refinement at the porous sample interfaces, was employed after verifying that it fulfills mesh convergence. The default solver was used.

Simulations were performed for the values of $k, b$, and $\varepsilon$ of cases 7-12 reported in Table II. The permeability was taken as to account for Klinkenberg effects according to the relationships given in Eqs. (5) and (38).

The range of intrinsic permeability was chosen in agreement with the fact that such an experiment is dedicated to poorly permeable materials. For $k_{l}>10^{-16} \mathrm{~m}^{2}$, the time to reach final equilibrium is on the order of a second or smaller, which is too short to carry out an experiment. As in the $1 \mathrm{D}$ case, $D$ and $\varepsilon$ were estimated in the least squares sense using an inverse procedure with the simplified linearized model [Eq. (32)], and $k$ was computed as $k=D \varepsilon$. The corresponding values, $D_{e s t}, \varepsilon_{e s t}$, and $k_{\text {est }}$, together with the relative errors, are reported in Table II.

As in the $1 \mathrm{D}$ case, the relative errors resulting from the linear approximation remain small. The maximum error on $\varepsilon_{\text {est }}$ is $2.8 \%$ and, as expected, is observed for the largest value of $\varepsilon$ (i.e., $\varepsilon=0.9$, case 8 ) combined to the largest value of $k_{l}$ under investigation here.
Moreover, the largest relative error on $k_{e s t}$, which remains, however, smaller than $4.3 \%$, is also observed in that case. Relative errors on $D_{\text {est }}$ are also very small, less than $1.4 \%$, over the whole range of parameters, confirming the validity of the linearized model. As already mentioned for the $1 \mathrm{D}$ configuration, $D$ is better estimated than $\varepsilon$, and the error on $k_{\text {est }}$ results from the cumulated errors on $D_{\text {est }}$ and $\varepsilon_{\text {est }}$.

\section{EXPERIMENTS}

\section{A. Materials and method}

The device used for permeability (and porosity) measurement of parallelepipedic samples is composed of the two tight tanks 0 and 1 of respective volumes $V_{0}$ and $V_{1}$ machined in a single massive aluminum part ensuring a uniform and constant temperature of the whole device. The two volumes are connected by the valve $V_{1}$ normally closed. Pressures in the two volumes are measured with Keller LEO3 pressure transducers having a range of $0-4$ bars and a precision of $0.1 \%$. The pressure transducers are connected to a data acquisition system ALMEMO 2290-4, which records pressures at a time-rate of $0.1 \mathrm{~s}$. Tank 0 is connected to a pressurized bottle (HP) of dry air used for the probing gas, and tank 1 is connected to the atmosphere via a normally closed valve that is used to bring the system to atmospheric pressure and flush it with dry air prior to an experiment. A picture of the device is shown in Fig. 8.

Assuming the ideal gas law for dry air in the experimental conditions, a temperature variation $\Delta T$ induces a pressure variation $\Delta P$ in the closed system such that $\frac{\Delta P}{P}=\frac{\Delta T}{T}$. For a pressure of $10^{5} \mathrm{~Pa}$ and a temperature of $293 \mathrm{~K}$, this indicates that $\Delta P=34 \mathrm{~Pa}$ (respectively, $340 \mathrm{~Pa}$ ) for $\Delta T=0.1 \mathrm{~K}$ (respectively, $1 \mathrm{~K}$ ). This suggests that the sample and the experimental device must be at thermal equilibrium and kept at a constant temperature for an accurate measurement. For this reason, the whole system is placed in a climatic enclosure Binder KBF115 to ensure a perfectly constant temperature over the measurement period.

The device used for permeability (and porosity) measurement of cylindrical samples of circular cross section is presented in Fig. 9.

TABLE II. Parameters used for the simulations and results of the estimated values in the $2 \mathrm{D}$ axisymmetric case.

\begin{tabular}{|c|c|c|c|c|c|c|c|}
\hline Case No. & & 7 & 8 & 9 & 10 & 11 & 12 \\
\hline$k_{l}$ & $\mathrm{~m}^{2}$ & $10^{-16}$ & $10^{-16}$ & $10^{-18}$ & $10^{-18}$ & $10^{-20}$ & $10^{-20}$ \\
\hline B & $\mathrm{Pa}$ & $1.087 \cdot 10^{5}$ & $1.087 \cdot 10^{5}$ & $5.71 \cdot 10^{5}$ & $5.71 \cdot 10^{5}$ & $29.95 \cdot 10^{5}$ & $29.95 \cdot 10^{5}$ \\
\hline$\varepsilon$ & & 0.05 & 0.9 & 0.05 & 0.2 & 0.05 & 0.2 \\
\hline$P_{c}$ & $\mathrm{~Pa}$ & $1.228 \cdot 10^{5}$ & $1.112 \cdot 10^{5}$ & $1.208 \cdot 10^{5}$ & $1.167 \cdot 10^{5}$ & $1.208 \cdot 10^{5}$ & $1.180 \cdot 10^{5}$ \\
\hline$\varepsilon_{e s t}$ & $\cdots$ & 0.0510 & 0.875 & 0.050 & 0.200 & 0.050 & 0.200 \\
\hline$\frac{\varepsilon_{e s t}-\varepsilon}{\varepsilon}$ & $\%$ & 2.0 & -2.8 & 0 & 0 & 0 & 0 \\
\hline$k^{\varepsilon}\left[\right.$ Eq. (5) with $\left.P=P_{c}\right]$ & $\mathrm{m}^{2}$ & $1.886 \cdot 10^{-16}$ & $1.978 \cdot 10^{-16}$ & $5.725 \cdot 10^{-18}$ & $5.891 \cdot 10^{-18}$ & $2.580 \cdot 10^{-19}$ & $2.639 \cdot 10^{-19}$ \\
\hline$k_{\text {est }}$ & $\mathrm{m}^{2}$ & $1.939 \cdot 10^{-16}$ & $1.896 \cdot 10^{-16}$ & $5.724 \cdot 10^{-18}$ & $5.867 \cdot 10^{-18}$ & $2.578 \cdot 10^{-19}$ & $2.637 \cdot 10^{-19}$ \\
\hline$\frac{k_{e s t}-k}{k}$ & $\%$ & 2.8 & -4.3 & -0.0 & -0.4 & -0.1 & -0.1 \\
\hline$D^{k}\left[\right.$ Eq. (5) with $\left.P=P_{c}\right]$ & $\mathrm{m}^{2}$ & $3.772 \cdot 10^{-15}$ & $2.197 \cdot 10^{-16}$ & $1.145 \cdot 10^{-16}$ & $2.946 \cdot 10^{-17}$ & $5.157 \cdot 10^{-17}$ & $1.319 \cdot 10^{-18}$ \\
\hline$D_{\text {est }}$ & $\mathrm{m}^{2}$ & $3.801 \cdot 10^{-15}$ & $2.166 \cdot 10^{-16}$ & $1.144 \cdot 10^{-16}$ & $2.938 \cdot 10^{-17}$ & $5.159 \cdot 10^{-17}$ & $1.320 \cdot 10^{-18}$ \\
\hline$\frac{D_{\text {est }-D}}{D}$ & $\%$ & -0.8 & -1.4 & -0.1 & -0.3 & 0.0 & 0.1 \\
\hline
\end{tabular}




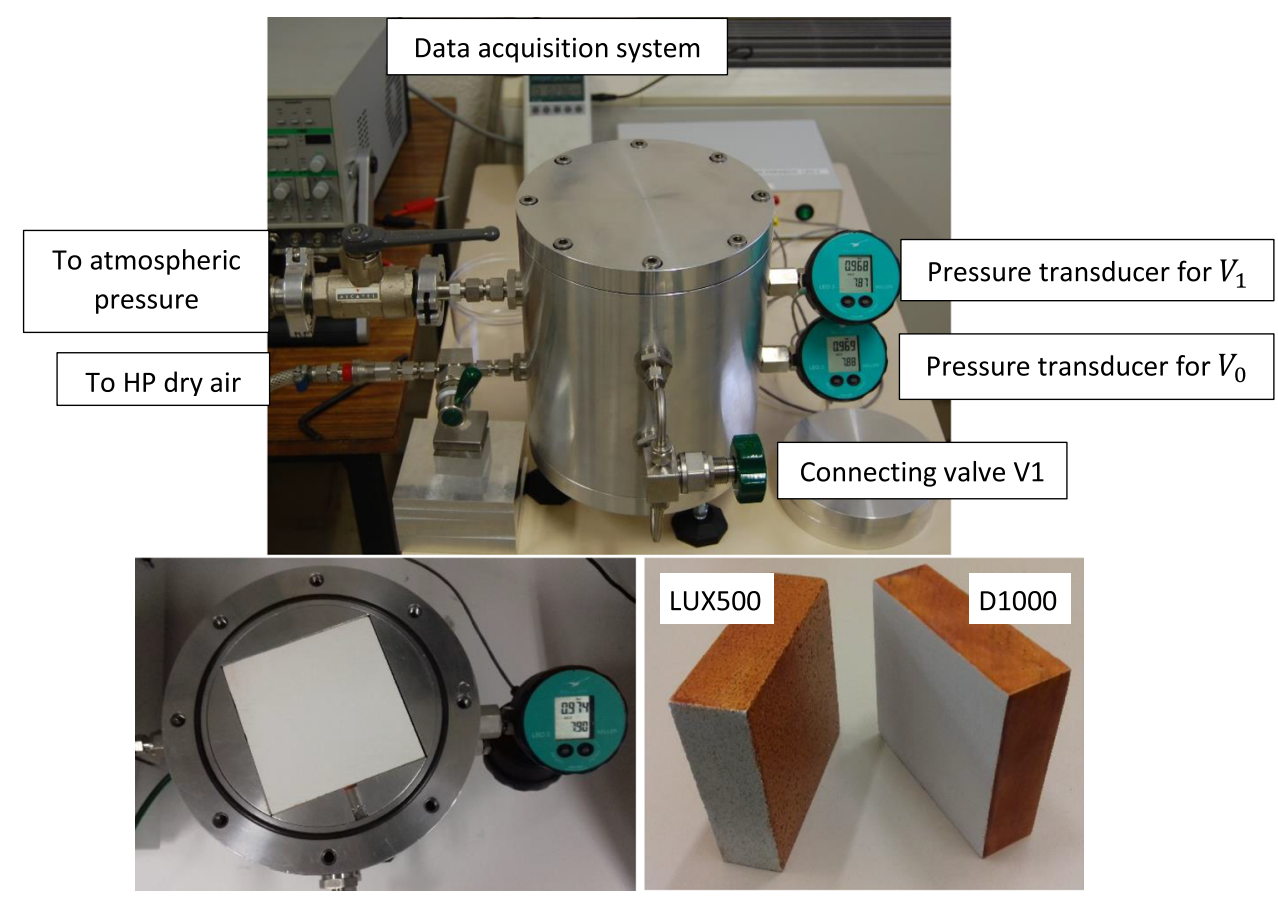

FIG. 8. Experimental device for parallelepipedic samples and samples used in the experiments (LUX500 and D1000).

\section{Measurement of volume $V_{1}$}

For both devices, the volume $V_{1}$ was determined prior to any experiment on a porous material. For this purpose, two experiments were carried out, the first one $(i=1)$ with both tanks empty and the second one $(i=2)$ with a piece of non-porous material (metal) of volume $V_{2}$ placed in tank 1 . A careful measurement of the metal piece dimensions was performed, allowing to determine $V_{2}$ accurately. For the two experiments ( 1 and 2 ), tank 0 was initially filled with a gas under a pressure $P_{0 i}$ and then connected to tank 1 initially filled with the same gas under pressure $P_{1 i}$. The final equilibrium pressure, $P_{f}$, was then measured. The protocol is schematically represented in Fig. 10.

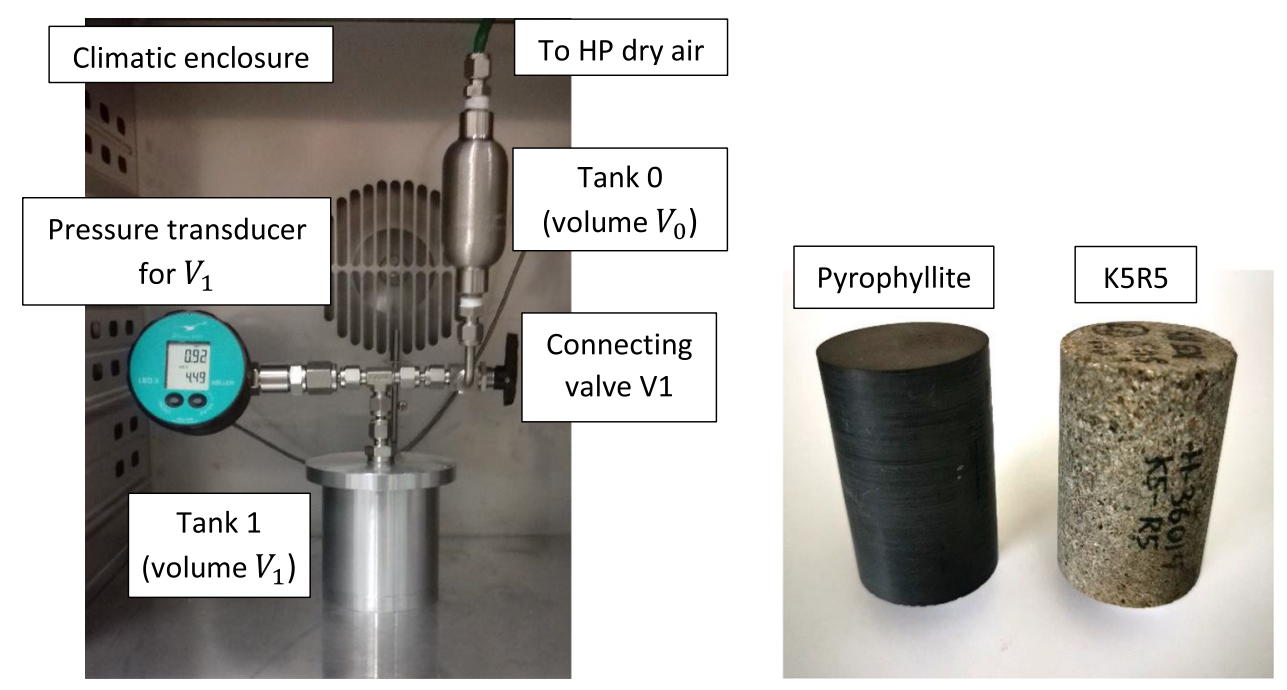

FIG. 9. Experimental device used for cylindrical samples and samples used in the experiments (pyrophyllite and K5R5). 


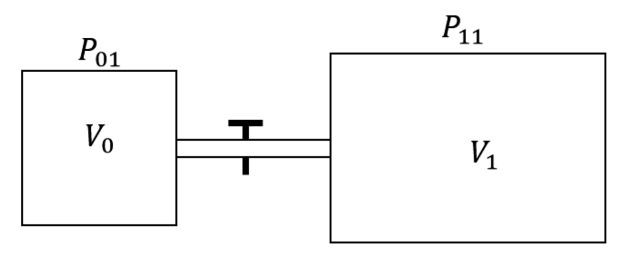

$1^{\text {st }}$ experiment: Final pressure $P_{f 1}$

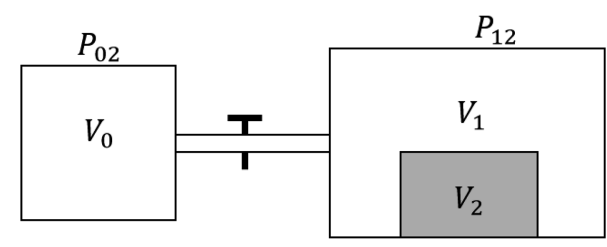

$2^{\text {nd }}$ experiment: Final pressure $P_{f 2}$

FIG. 10. Schematic representation of the two experiments performed to determine volumes $V_{1}$ (and $V_{0}$ ).

From the mass balance equations between the two stages of these two experiments, the volume $V_{1}$ can be determined as

$$
V_{1}=\frac{\left(P_{01}-P_{f 1}\right)\left(P_{f 2}-P_{12}\right)}{\left(P_{01}-P_{f 1}\right)\left(P_{f 2}-P_{12}\right)+\left(P_{f 2}-P_{02}\right)\left(P_{f 1}-P_{11}\right)} V_{2} .
$$

Note that $V_{0}$ can also be obtained as

$$
V_{0}=\frac{\left(P_{f 1}-P_{11}\right)\left(P_{f 2}-P_{12}\right)}{\left(P_{01}-P_{f 1}\right)\left(P_{f 2}-P_{12}\right)+\left(P_{f 2}-P_{02}\right)\left(P_{f 1}-P_{11}\right)} V_{2} .
$$

\section{Experimental protocol}

The dynamic experiment, aiming at the determination of $k$ (and $\varepsilon)$, is carried out as follows: The sample is placed in tank 1 , which is sealed with the cap. While the valve $V_{1}$ is closed, the pressure of dry air is raised in tank 0 to $P_{i}+\Delta P$. The pressure recording in tank 1 $\left[P_{1}(t)\right]$ is started $(t=0)$ and $V_{1}$ is opened and closed shortly after $\left(t=t_{c}\right)$ in order to create a pressure pulse in tank 1 . The value of $\Delta P$ is set to a value so as to create a pressure increment in tank 1 of about $3 \cdot 10^{4} \mathrm{~Pa}$. It can be predicted from the knowledge of $V_{0}$ and $V_{1}$. The recording of $P_{1}(t)$ ends when no noticeable variation is observed, corresponding to equilibrium at $t=t_{f}$.

At this stage, $V_{1}$ is opened and the pressure is eventually adjusted to the desired initial value. After a new equilibrium is reached, $V_{1}$ is closed. A new pressure increment $\Delta P$ is set in tank 0 and pressure recording is started in tank 1 . The valve $V_{1}$ is open and rapidly closed. The recording of $P_{1}(t)$ is stopped when equilibrium is reached again. The procedure is repeated as many times as desired, featuring different values of $P_{c}$ whose expression is given in Eq. (9). In this way, the dependence of $k$ on pressure due to Klinkenberg effects can be investigated, allowing the determination of the Klinkenberg coefficient, making use of the relationship in Eq. (5). In the present work, it was repeated until the initial pressure reaches $3.5 \cdot 10^{5} \mathrm{~Pa}$. A lower bound constraint for the time interval of pressure recordings used for the estimation was fixed to $2 \mathrm{~s}$, a value that typically corresponds to the time required to reach pressure equilibrium after a pressure pulse is set in tank 1 in the absence of a porous sample. In other words, the two first seconds of pressure recordings after valve opening were not considered in the inverse procedure.

\section{B. Results in the 1D case: LUX500 and D1000}

The volume $V_{1}$ was measured according to the procedure described in Sec. IV A 1 yielding $V_{1}=3.74 \times 10^{-4} \mathrm{~m}^{3}$. Two different insulating materials, namely, LUX500 and D1000, were employed (see Fig. 8). The dimensions of the LUX500 and D1000 samples were $99.5 \times 99.5 \times 30 \mathrm{~mm}^{3}$, all the faces being sealed except one of section $99.5 \times 30 \mathrm{~mm}^{2}$ for the former, which means $L=99.5 \mathrm{~mm}$, whereas the open section for the latter was $99.5 \times 99.5 \mathrm{~mm}^{2}$, i.e., $L=30 \mathrm{~mm}$. Prior to dynamic experiments, $\varepsilon$ was determined from pycnometry tests, yielding $\varepsilon=0.68$ for LUX500 and $\varepsilon=0.48$ for D1000. Dynamic experiments were then carried out, and the inverse procedure was run on the recorded signals of $P_{1}(t)$, making use of the linearized model given in Eq. (18) in order to determine $D$ from Eq. (36). The porosity was also estimated from this procedure using Eq. (37). Note that $X_{1}$ [see Eq. (19)] was also estimated, albeit of no special interest regarding the material characterization. The permeability, $k$, was computed as $k=D \varepsilon$, taking the value of $\varepsilon$ obtained from the pycnometry experiments.

Examples of pressure recordings, $P_{1 \exp }$, together with the corresponding signals, $P_{1 m o d}$, computed with the optimal estimated values of $D$ and $\varepsilon$ are reported in Figs. 11(a) and 11(c) for the LUX500 and D1000 samples, respectively. In both cases, the two signals are perfectly superimposed, showing the excellent performance of the identification process and the relevance of the estimations. This is confirmed by the residues, $P_{1 \exp }-P_{1 \text { mod }}$, amplified by a factor of 10 and also reported in these figures. For both materials, the residues are on the order of $5 \times 10^{3} \mathrm{~Pa}$ at the early stage of the experiment and rapidly decrease, after few seconds, down to around $10^{3} \mathrm{~Pa}$ (LUX500) and $1.6 \cdot 10^{3} \mathrm{~Pa}$ (D1000). They remain very small compared to the amplitude of $P_{1}$ and well centered on zero. In Figs. 11(b) and $11(\mathrm{~d})$, the reduced sensitivity of $P_{1}$ to $D$ is represented vs the reduced sensitivity to $\varepsilon$, respectively, for the LUX500 and D1000 materials. These graphs clearly show that the two sensitivities are not proportional, justifying that the two parameters are uncorrelated and can therefore be estimated from the inverse procedure for both materials. This confirms the relevance of the estimation.

Six different experiments were carried out at different values of $P_{c}$ ranging from $1.17 \cdot 10^{5}$ to $3.67 \cdot 10^{5} \mathrm{~Pa}$, both for the LUX500 and D1000 samples, and each experiment was repeated three times The variations of $k$ with respect to $\frac{1}{P_{c}}$ are reported in Fig. 12(a) (LUX500) and Fig. 12(c) (D1000) along with the standard deviations computed from the three distinct tests. These graphs show that the apparent permeability can be satisfactorily correlated with $\frac{1}{P_{c}}$ with a linear fit, confirming that $k$ follows a Klinkenberg relationship. The linear correlations lead to an intrinsic permeability, $k_{l}$, and a Klinkenberg coefficient, $b$, whose values are reported in Table III.

The values of porosity estimated from six different experiments are represented in Figs. 12(b) and 12(d) vs $P_{c}$ for the LUX500 and D1000 samples, respectively. The standard deviations on the three 
a)

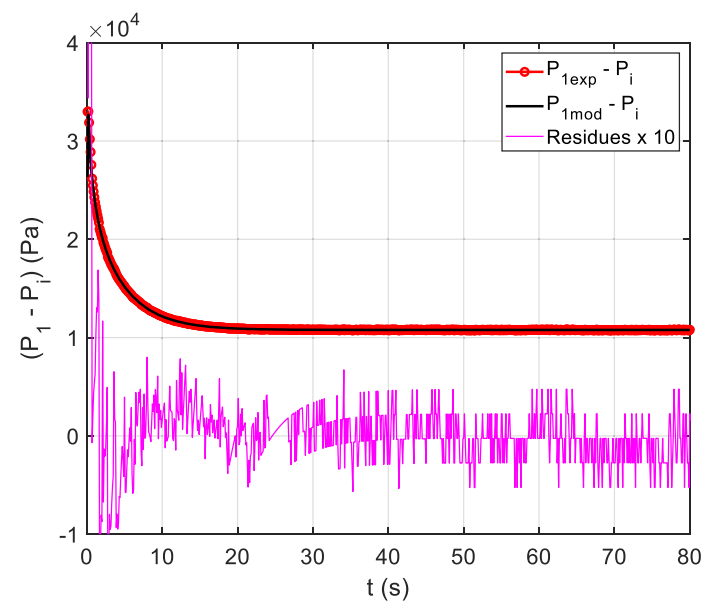

c)

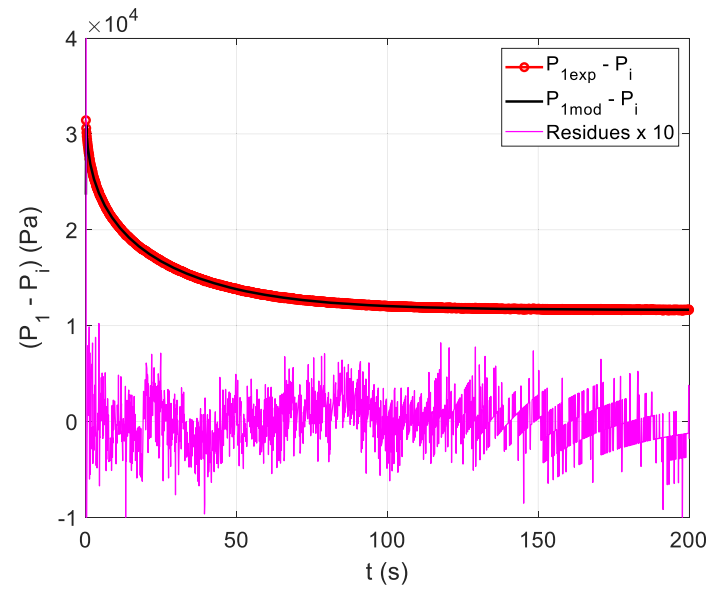

b)

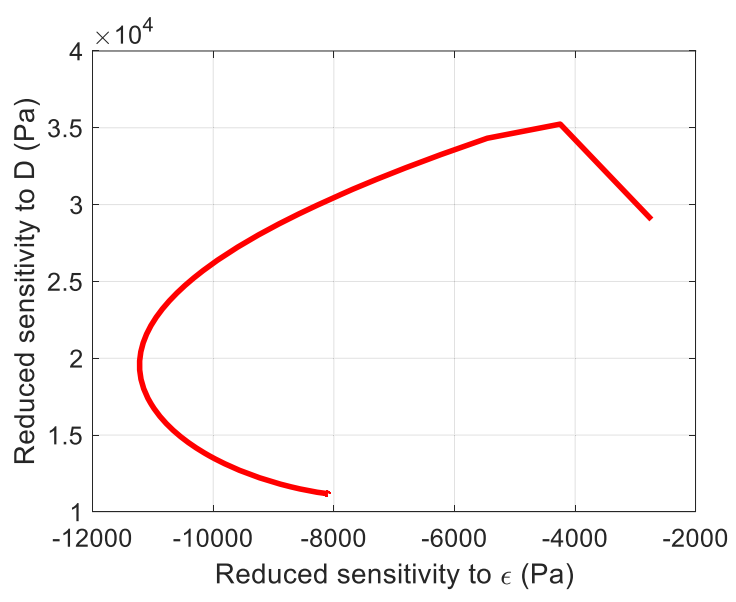

d)

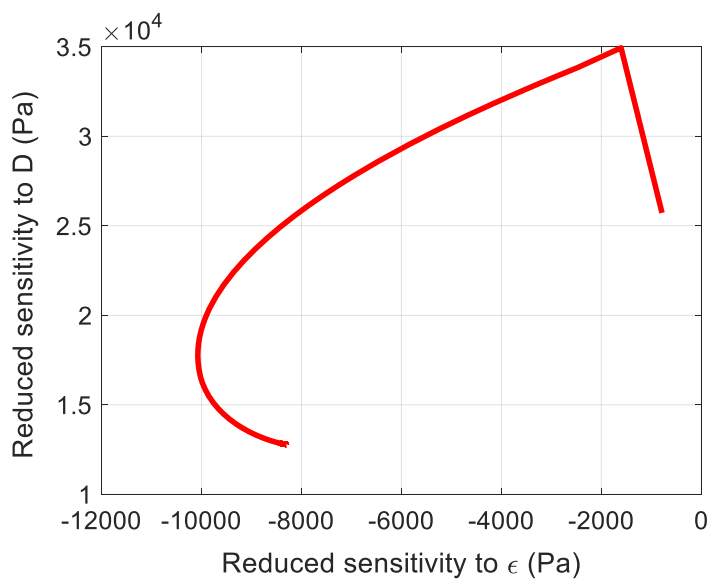

FIG. 11. (a) and (c) Pressure evolutions in tank 1 obtained experimentally $\left(P_{1 \text { exp }}\right)$ and simulated with the optimal estimated parameters $\left(P_{1 \bmod }\right)$. Residues $\left(P_{1 \text { exp }}-P_{1 \text { mod }}\right)$ are represented with an amplification factor of 10 . (a) $P_{i}=3.5 \cdot 10^{5} \mathrm{~Pa}$. (c) $P_{i}=210^{5} \mathrm{~Pa}$. (b) and (d) Reduced sensitivity to $D$ vs reduced sensitivity to $\varepsilon$. (a) and (b) LUX 500. (c) and (d) D1000.

tests performed at each value of $P_{c}$ are also reported in these figures. The maximum relative difference on these estimated values, taking the average value as the reference, is 7.3\% (LUX500) and 3\% (D1000).

The average values are indicated in Table III and are very close to the values obtained from the pycnometry measurements, the relative difference being around $0.6 \%$ and $1 \%$ for the LUX500 and D1000 samples, respectively.

\section{Results in the 2D axisymmetric configuration}

Using the same method as in the 1D case, the volume of tank 1 was measured to be $V_{1}=7.85 \times 10^{-5} \mathrm{~m}^{3}$. Two different materials, a rock denoted K5R5 and a pyrophyllite, were tested using cylindrical samples (see Fig. 9), which were $38.0 \mathrm{~mm}$ in diameter and 60.0 $\mathrm{mm}$ in length, with all faces left open. Experiments were performed following the protocol described in Sec. IV A 2. The parameters $D$ and $\varepsilon$ (as well as $X_{1}$ ) were estimated by making use of the inverse procedure with the model given in Eq. (32) in which the series was evaluated keeping 200 terms to ensure convergence. The permeability, $k$, was determined from $k=D \varepsilon$ with $\varepsilon$ obtained from the pycnometry experiments.

\section{Rock K5R5}

The porosity measured with the pycnometry experiment is $\varepsilon=$ 0.051 . Dynamic experiments were carried out at eight different values of $P_{c}$ ranging between $0.35 \cdot 10^{5}$ and $3.8 \cdot 10^{5} \mathrm{~Pa}$. Only one test was performed for each experiment.

Estimated values of the apparent permeability corresponding to each value of $P_{c}$ are represented in Fig. 13(a) vs $\frac{1}{P_{c}}$. These results show that the dependence of $k$ on $P_{c}$ can be interpreted with a Klinkenberg relationship [Eq. (5)]. A linear regression on the data 
a)

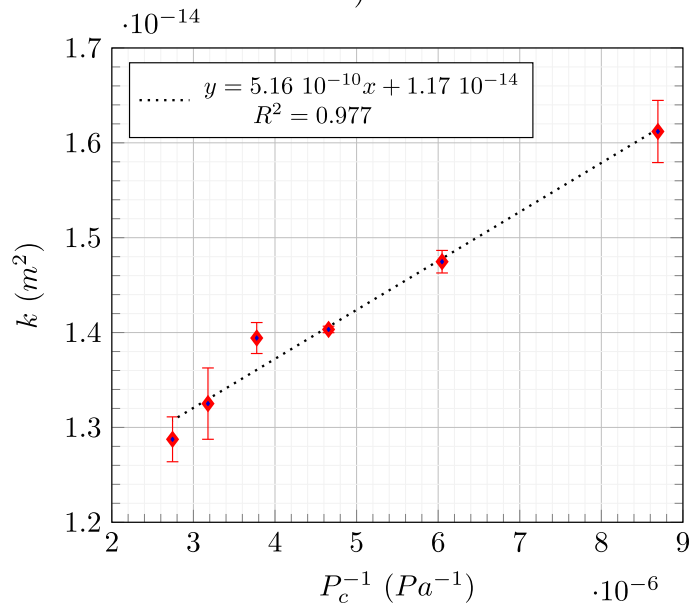

c)

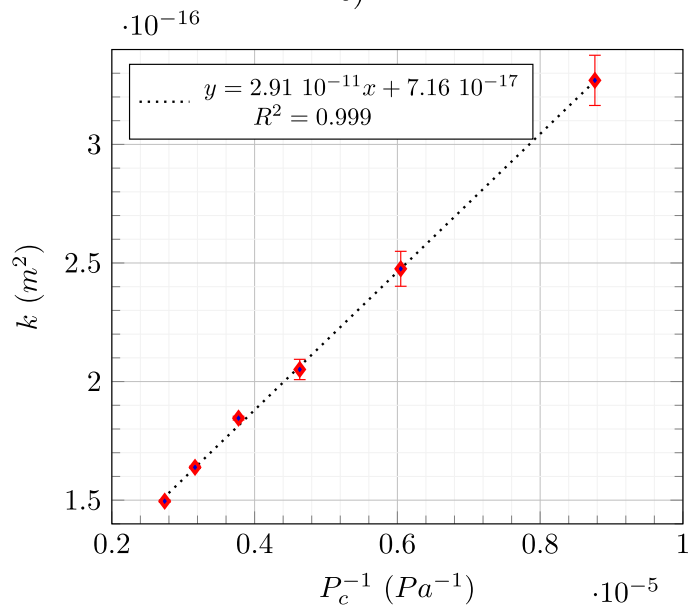

b)

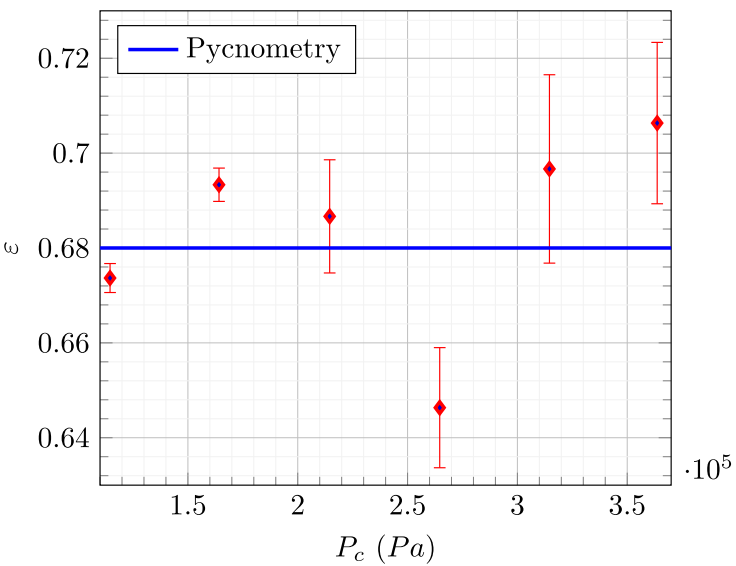

d)

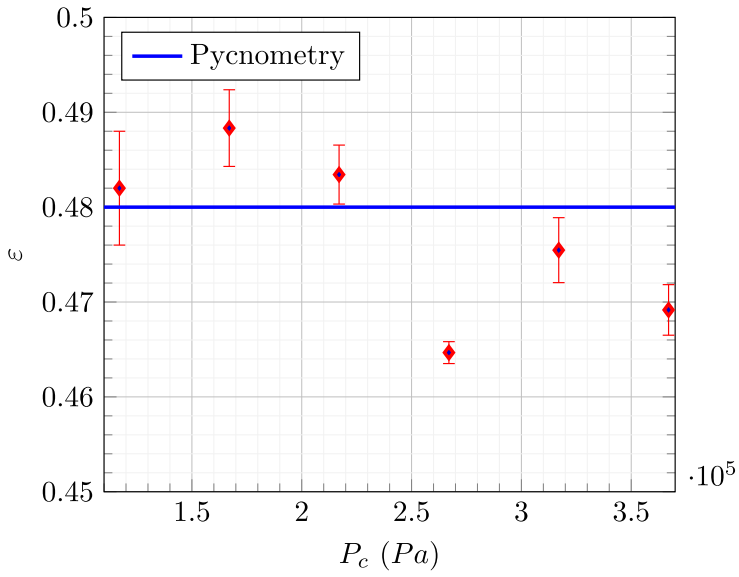

FIG. 12. (a) and (c) Estimated values of the apparent permeability, $k$, obtained from six different experiments, each of them repeated three times, carried out at different values of $P_{c}$ vs $\frac{1}{P_{c}}$. The error bars correspond to the standard deviations computed from the three tests. The linear regressions agree with a Klinkenberg relationship. (b) and (d) Estimated values of $\varepsilon$ and error bars corresponding to the standard deviations obtained from the six experiments. Solid lines materialize the porosity values obtained from pycnometry experiments. (a) and (b) LUX500. (c) and (d) D1000.

points of Fig. 13(a) leads to $b=3.95 \cdot 10^{5} \mathrm{~Pa}$ and an intrinsic permeability $k_{l}=3.49 \cdot 10^{-17} \mathrm{~m}^{2}$. In Fig. 13(b), the estimated values of the porosity obtained from the six experiments are represented vs $P_{c}$. The average value is $\varepsilon=0.0506$, which gives an error of less than

TABLE III. Results on the intrinsic permeability, Klinkenberg coefficient, and porosity obtained from the estimation using the inverse procedure within the range of $P_{c}$ considered in the experiments. The values of $\varepsilon$ determined from pycnometry experiments are also recalled. LUX500 and D1000.

\begin{tabular}{lcc}
\hline \hline Material & LUX500 & D 1000 \\
\hline$P_{c}$ range $(\mathrm{Pa})$ & $1.17 \cdot 10^{5}-3.67 \cdot 10^{5}$ & $1.17 \cdot 10^{5}-3.67 \cdot 10^{5}$ \\
$k_{l}\left(\mathrm{~m}^{2}\right)$ & $1.17 \cdot 10^{-14}$ & $7.16 \cdot 10^{-17}$ \\
$b(\mathrm{~Pa})$ & $0.44 \cdot 10^{5}$ & $4.06 \cdot 10^{5}$ \\
$\varepsilon$ (average) & 0.684 & 0.475 \\
$\varepsilon$ (pycnometry) & 0.68 & 0.48 \\
\hline \hline
\end{tabular}

$0.8 \%$ with respect to the value obtained from pycnometry, taking the latter as the reference. The maximum difference on the six estimated values is $3 \%$, taking the average as the reference.

\section{Pyrophyllite}

The pycnometry test was first performed on the pyrophyllite sample yielding $\varepsilon=0.048$. Six dynamic experiments were then carried out in a range of $P_{c}$ between $0.38 \cdot 10^{5}$ and $2.18 \cdot 10^{5} \mathrm{~Pa}$. Only one test was performed at each value of $P_{c}$.

The estimated values of $k$ are represented in Fig. 14(a) vs $\frac{1}{P_{c}}$. Although they seem to obey a linear correlation, these results cannot be interpreted by a Klinkenberg relationship, as given in Eq. (5), which, indeed, would lead to unphysical negative values of $k_{l}$ and $b$. The porosity, estimated from the dynamic experiments, is reported in Fig. 14(b) yielding an average value $\varepsilon=0.0465$, a maximum relative difference over the six values of about $8 \%$, and a relative error 
a)

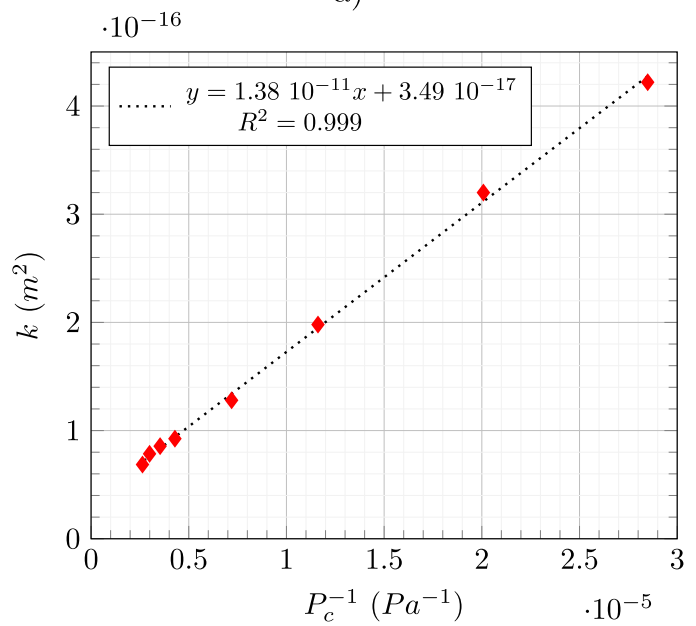

b)

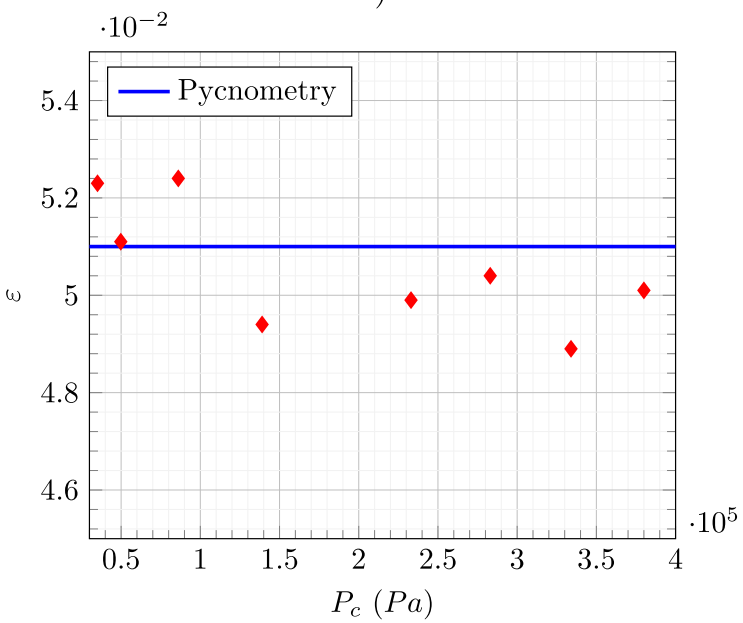

FIG. 13. K5R5: (a) estimated values of $k$ obtained from experiments carried out at eight different values of $P_{c}$ and (b) estimated values of $\varepsilon$ from the eight experiments performed at different values of $P_{c}$. The solid line represents the value of $\varepsilon$ obtained from pycnometry.

with respect to the value obtained from pycnometry of about $3 \%$. For this sample, it must be pointed out, however, that the estimated values of $k$ and $\varepsilon$ depend on the interval of time-recording of $P_{1 \exp }$ considered for the inverse procedure. Moreover, as shown in Fig. 15(a), representing $P_{1 \exp }$ for an experiment at $P_{i}=0.93 \cdot 10^{5}$ $\mathrm{Pa}$ and the corresponding $P_{1 \text { mod }}$ obtained with the estimated values of $k$ and $\varepsilon$ considering $P_{1 \exp }$ up to $3000 \mathrm{~s}$ in the inverse procedure, the experimental pressure decrease is not captured by the model for $t>3000 \mathrm{~s}$, yielding residues that are not well centered on zero. This is further illustrated in Fig. 15(b) in which the estimated values of $k$ and $\varepsilon$ are represented vs the upper bound of the time interval employed in the inverse procedure to evaluate them. This figure shows that $k$ decreases while $\varepsilon$ increases when a longer recording of $P_{1 \exp }$ is considered in the estimation procedure. Nevertheless, these variations remain moderate. In fact, taking the values estimated with the upper bound of the time-recording of $P_{1 \exp }$ equal to 4000 s shows that $k$ and $\varepsilon$ do not vary by more than around $20 \%$ and $3 \%$, respectively, for all the other time intervals considered for the estimation.

These results are consistent with the idea that this clay-rich material is heterogeneous and/or anisotropic with the presence of micro-fissures. The assessment of this conjecture would require further investigations with more sophisticated means that are beyond the scope of the present work. This example illustrates the a)

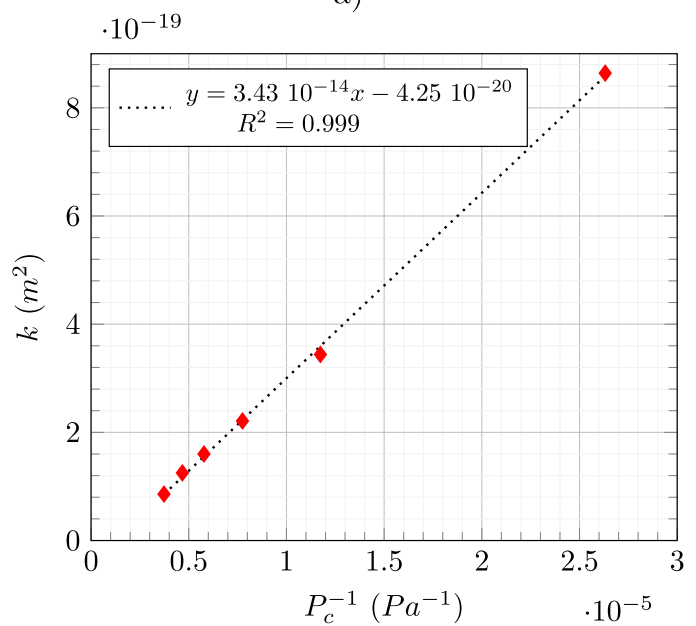

b)

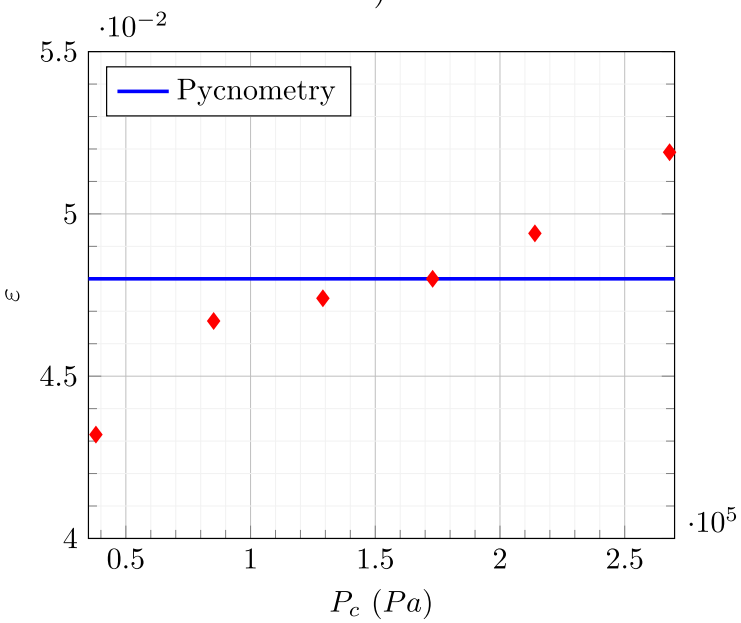

FIG. 14. Pyrophyllite: (a) estimated values of $k$ obtained from experiments carried out at six different values of $P_{c}$ and (b) estimated values of $\varepsilon$ from the six experiments performed at different values of $P_{c}$. The solid line represents the value of $\varepsilon$ obtained from pycnometry. 
a)

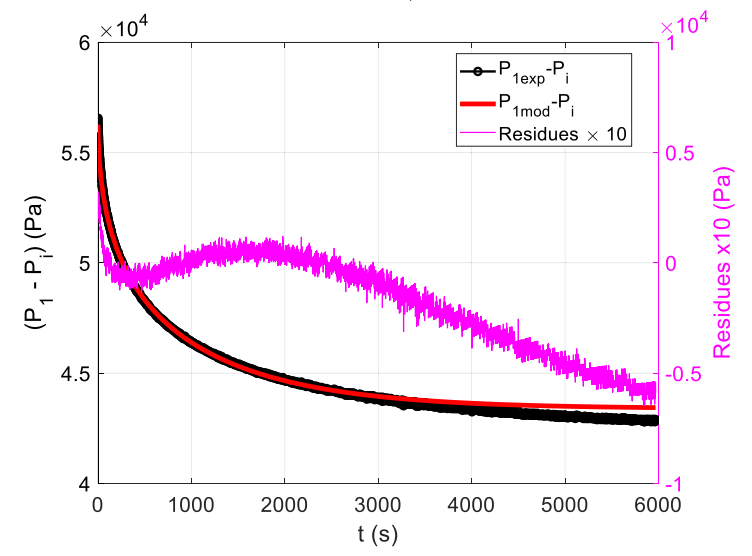

b)

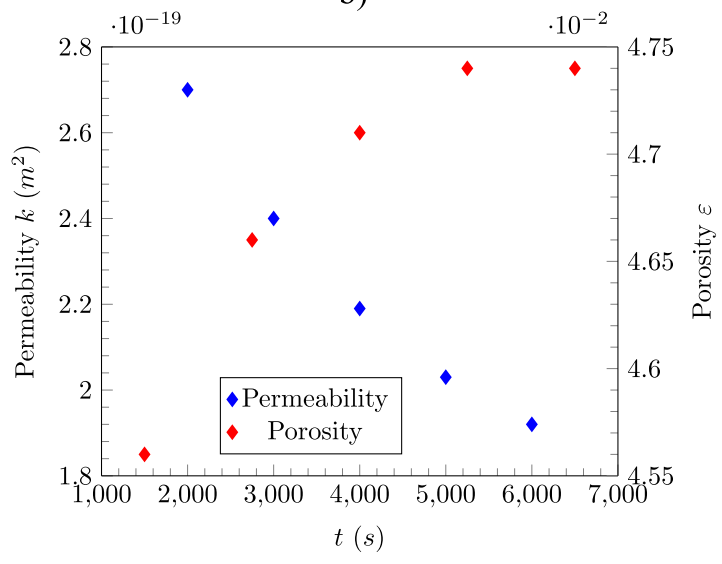

FIG. 15. (a) Experimental pressure evolution, $P_{1 \exp }$, and predicted signal, $P_{1 \text { mod }}$, from the model [Eq. (32)] with the values of $k$ and $\varepsilon$ estimated on $P_{1 \text { exp }}(t)$ up to $3000 \mathrm{~s}$. The residues $(\times 10)$ decrease beyond $t=3000 \mathrm{~s}$ at which the model predicts quasi-equilibrium. (b) Estimated values of $k$ and $\varepsilon$ vs the interval of time-recording of $P_{1 \text { exp }}$ considered for the estimation. $P_{c}=1.29 \cdot 10^{5} \mathrm{~Pa}$.

potential capability of the procedure developed in this work to diagnose non-homogeneous and/or non-isotropic permeable porous materials.

\section{CONCLUSIONS}

New developments of the GRI method to determine the permeability of porous media (together with the porosity and Klinkenberg coefficient), widely extending the technique far beyond its classical use on crushed porous materials, are proposed in this work. They allow one to carry out measurements on samples having a cylindrical shape. In both cases, a quasi-analytical solution is provided when the probing gas flow is in one direction, assuming that it corresponds to a principal direction of the permeability tensor. Quasianalytical solutions are also developed for a three-dimensional flow (parellelipidedic sample) and a two-dimensional axisymmetric flow (cylindrical sample of circular cross section) with the hypothesis that the material is homogeneous and isotropic. A general hypothesis is that the flow during measurement is weakly compressible, allowing to treat the probing gas compressibility as a constant. These solutions are employed to interpret the experimental pressure recording by solving an inverse problem. The validity of the method, together with the relevance of the constant compressibility assumption, were checked over a wide range of permeability by a sensitivity analysis and a comparison with the results obtained from direct simulations of the complete non-linear initial boundary value problem. The efficiency of the experimental protocol and interpretation procedure were illustrated with experiments on four different porous materials featuring permeabilities in the range $10^{-14}$ to $10^{-19} \mathrm{~m}^{2}$ in the one-dimensional and axisymmetric configurations. The potential capability of diagnosing heterogeneous and/or anisotropic materials was highlighted on a pyrophyllite sample. Moreover, repeating the experiment in the $1 \mathrm{D}$ and 3D configurations on the same sample would provide an efficient way of diagnosing anisotropy and/or inhomogeneity of the porous material under concern.
The method reported here has the advantage of requiring a simple experimental device. Some issues of efficient sealing and/or unwanted stress effects associated with the sample confinement required in many other experimental methods are circumvented. It is fast and can be applied over a very wide range of permeability values. It allows the simultaneous determination of the permeability, porosity, and Klinkenberg coefficient when slip effects may be present. However, it is restricted to measurements on homogeneous samples.

As a matter of perspective, a similar methodology could be further developed to the case of a (homogeneous) sample of arbitrary shape. This could be achieved by first determining the shape and dimensions of the sample and applying the inverse procedure by making use of a direct numerical simulation of the mass and momentum governing equations with the appropriate boundary and initial conditions. This would also allow one to remove the hypothesis of a constant compressibility used in the present work while considering larger pressure variations during the measurements. As a further extension, one may also consider this procedure to be applied to the estimation of the effective (or relative) permeability of a porous material partially saturated with another (wetting) fluid. This could be envisaged, provided that gas flow during the measurement would not induce any evaporation (which would otherwise produce a probing gas pressure perturbation) and would not lead to a redistribution of the fluid in place.

\section{APPENDIX A: SOLUTION FOR A PARALLELEPIPEDIC SAMPLE}

In this appendix, the main steps to derive the solution for the pressure evolution in tank 1 , when a parallelepipedic sample is used with all its faces open to gas flow, are provided. The sample is supposed to be of size $\ell, L$, and $h$ in the $x, y$, and $z$ directions, respectively. Assuming the origin at the center of the sample and assuming, as in the $1 \mathrm{D}$ case, that $P$ experiences small variations compared to $P_{c}$ [see Eq. (9)], the initial boundary value problem can be stated as follows: 


$$
\frac{\partial^{2} P}{\partial x^{2}}+\frac{\partial^{2} P}{\partial y^{2}}+\frac{\partial^{2} P}{\partial z^{2}}=\frac{1}{a} \frac{\partial P}{\partial t}
$$

B.C. $1 \quad \frac{\partial P}{\partial x}=0 \quad$ at $x=0$,

B.C.2

$$
\frac{\partial P}{\partial y}=0 \quad \text { at } y=0
$$

B.C.3

$$
\frac{\partial P}{\partial z}=0 \quad \text { at } z=0
$$

B.C. 4

$$
P=P_{1}(t) \quad \text { at } x=\frac{\ell}{2},
$$

B.C.5

$$
P=P_{1}(t) \quad \text { at } y=\frac{L}{2},
$$

B.C.6

$$
P=P_{1}(t) \quad \text { at } z=\frac{h}{2},
$$

I.C.

$$
P(x, 0)=P_{i} \quad \text { at } t=0 .
$$

In Eq. (A1), $a$ is given by Eq. (11), i.e., $a=\frac{k}{\varepsilon \mu \beta}$. Boundary conditions in Eqs. (A2)-(A4) express the fact that the fluxes are zero at the midplanes in the sample as a result of symmetry.

To complete the model, the gas mass flux balance at $t$ in the system including tank 1 and the sample can be expressed by writing

$$
\frac{M}{R T}\left(V_{1}-V\right) \frac{d P_{1}}{d t}=-\varepsilon V \frac{M}{R T} \frac{d P_{m}}{d t}+\dot{q}_{m 0}
$$

Here, $P_{m}$ denotes the mean pressure within the sample at $t$ [see Eq. (8)], whereas $\dot{q}_{m 0}$ is the flux of gas entering tank 1 to initiate the experiment.

At this stage, the problem is solved in the Laplace-Fourier domain. To do so, a Laplace transform, $\mathcal{L}$, is first applied to the above equations. Denoting $\theta=\mathcal{L}\left(P-P_{i}\right)$ and $\theta_{1}=\mathcal{L}\left(P_{1}-P_{i}\right)$ leads to

$$
\begin{array}{cc}
\frac{\partial^{2} \theta}{\partial x^{2}}+\frac{\partial^{2} \theta}{\partial y^{2}}+\frac{\partial^{2} \theta}{\partial z^{2}}=\frac{p}{a} \theta, \\
\frac{\partial \theta}{\partial x}=0 & \text { at } x=0, \\
\frac{\partial \theta}{\partial y}=0 & \text { at } y=0, \\
\frac{\partial \theta}{\partial z}=0 & \text { at } z=0, \\
\theta=\theta_{1}(p) & \text { at } x=\frac{\ell}{2},
\end{array}
$$

$$
\begin{aligned}
& \theta=\theta_{1}(p) \quad \text { at } y=\frac{L}{2}, \\
& \theta=\theta_{1}(p) \quad \text { at } z=\frac{h}{2} .
\end{aligned}
$$

A cosine Fourier transform, defined by $\bar{\theta}=\int_{0}^{\frac{\ell}{2}} \theta \cos \left(\alpha_{n} x\right) d x$, is now applied, yielding

$$
\frac{\partial^{2} \bar{\theta}}{\partial y^{2}}+\frac{\partial^{2} \bar{\theta}}{\partial z^{2}}-\alpha_{n}^{2} \bar{\theta}+\alpha_{n} \theta_{1} \sin \left(\frac{\alpha_{n} \ell}{2}\right)=\frac{p}{a} \bar{\theta},
$$

$$
\frac{\partial \bar{\theta}}{\partial y}=0 \quad \text { at } y=0
$$

$$
\begin{gathered}
\frac{\partial \bar{\theta}}{\partial z}=0 \quad \text { at } z=0, \\
\bar{\theta}=\frac{\theta_{1}}{\alpha_{n}} \sin \left(\frac{\alpha_{n} \ell}{2}\right) \quad \text { at } y=\frac{L}{2}, \\
\bar{\theta}=\frac{\theta_{1}}{\alpha_{n}} \sin \left(\frac{\alpha_{n} \ell}{2}\right) \quad \text { at } z=\frac{h}{2},
\end{gathered}
$$

where $\alpha_{n}$ are the eigenvalues given by $\alpha_{n}=\frac{(2 n-1) \pi}{\ell}$.

A second cosine Fourier transform, defined by $\overline{\bar{\theta}}=\int_{0}^{\frac{L}{2}} \bar{\theta} \cos \left(\gamma_{m} y\right) d y$, is again applied to the above equations to obtain

$$
\begin{aligned}
\frac{\partial^{2} \overline{\bar{\theta}}}{\partial z^{2}} & -\gamma_{m}{ }^{2} \bar{\theta}+\gamma_{m} \frac{\theta_{1}}{\alpha_{n}} \sin \left(\frac{\alpha_{n} \ell}{2}\right) \sin \left(\frac{\gamma_{m} L}{2}\right)-\alpha_{n}{ }^{2} \bar{\theta} \\
& +\alpha_{n} \theta_{1} \sin \left(\frac{\alpha_{n} \ell}{2}\right) \frac{1}{\gamma_{m}} \sin \left(\frac{\gamma_{m} L}{2}\right)=\frac{p}{a} \bar{\theta}
\end{aligned}
$$

or, equivalently,

$$
\begin{gathered}
\frac{\partial^{2} \bar{\theta}}{\partial z^{2}}-\left(\alpha_{n}{ }^{2}+\gamma_{m}{ }^{2}+\frac{p}{a}\right) \bar{\theta}+\theta_{1}\left(\frac{\alpha_{n}}{\gamma_{m}}+\frac{\gamma_{m}}{\alpha_{n}}\right) \sin \left(\frac{\alpha_{n} \ell}{2}\right) \\
\times \sin \left(\frac{\gamma_{m} L}{2}\right)=0, \\
\frac{\partial \theta}{\partial z}=0 \text { at } z=0, \\
\bar{\theta}=\frac{\theta_{1}}{\alpha_{n} \gamma_{m}} \sin \left(\frac{\alpha_{n} \ell}{2}\right) \sin \left(\frac{\gamma_{m} L}{2}\right) \text { at } z=\frac{h}{2} .
\end{gathered}
$$

Here, $\gamma_{m}$ are the eigenvalues, whose expression is $\gamma_{m}=\frac{(2 m-1) \pi}{L}$.

The solution to Eq. (A23) is

$$
\overline{\bar{\theta}}=A \cosh \left(\delta_{n m} z\right)+B \sinh \left(\delta_{n m} z\right)+C,
$$


with

$$
\delta_{n m}^{2}=\alpha_{n}^{2}+\gamma_{m}^{2}+\frac{p}{a}
$$

and

$$
C=\frac{\theta_{1}\left(\frac{\alpha_{n}}{\gamma_{m}}+\frac{\gamma_{m}}{\alpha_{n}}\right) \sin \left(\frac{\alpha_{n} \ell}{2}\right) \sin \left(\frac{\gamma_{m} L}{2}\right)}{\delta_{n m}^{2}} .
$$

Making use of the boundary conditions given in Eqs. (A24) and (A25), respectively, yields

$$
B=0
$$

and

$$
A=\frac{\theta_{1} \sin \left(\frac{\alpha_{n} \ell}{2}\right) \sin \left(\frac{\gamma_{m} L}{2}\right)\left[\frac{1}{\alpha_{n} \gamma_{m}}-\left(\frac{\alpha_{n}}{\gamma_{m}}+\frac{\gamma_{m}}{\alpha_{n}}\right) \frac{1}{\delta_{n m}^{2}}\right]}{\cosh \left(\delta_{n m} \frac{h}{2}\right)} .
$$

As a result, the solution in Eq. (A26) can be written as

$$
\begin{aligned}
\overline{\bar{\theta}}= & \theta_{1} \sin \left(\frac{\alpha_{n} \ell}{2}\right) \sin \left(\frac{\gamma_{m} L}{2}\right)\left\{\left[\frac{1}{\alpha_{n} \gamma_{m}}-\left(\frac{\alpha_{n}}{\gamma_{m}}+\frac{\gamma_{m}}{\alpha_{n}}\right) \frac{1}{\delta_{n m}{ }^{2}}\right]\right. \\
& \left.\times \frac{\cosh \left(\delta_{n m} z\right)}{\cosh \left(\delta_{n m} \frac{h}{2}\right)}+\frac{\left(\frac{\alpha_{n}}{\gamma_{m}}+\frac{\gamma_{m}}{\alpha_{n}}\right)}{\delta_{n m}{ }^{2}}\right\} .
\end{aligned}
$$

From this expression and the definition of the Fourier transform, the expression of $\theta$ is obtained as

$$
\theta=16 \sum_{n=1}^{\infty} \sum_{m=1}^{\infty} \frac{\overline{\bar{\theta}} \cos \left(\alpha_{n} x\right) \cos \left(\gamma_{m} y\right)}{\ell L} .
$$

A Laplace transform can now be applied to the flux relationship expressed in Eq. (A9), leading to

$$
\theta_{1}+\frac{\varepsilon V}{V_{1}-V} \theta_{m}=\frac{R T}{M\left(V_{1}-V\right)} \frac{\Phi_{1}}{p},
$$

in which $\theta_{m}$ and $\Phi_{1}$ are the Laplace transforms of, respectively, $P_{m}$ and $\dot{q}_{m 0}$. Keeping in mind that $P_{m}$ is the mean pressure in the sample allows one to write for $\theta_{m}$,

$$
\theta_{m}=\frac{8}{\ell L h} \int_{0}^{\frac{\ell}{2}} \int_{0}^{\frac{L}{2}} \int_{0}^{\frac{h}{2}} \theta \mathrm{d} x \mathrm{~d} y d z
$$

where $\theta$ is given by Eq. (A32). Performing the three integrations in the above equation yields

$$
\begin{aligned}
\theta_{m}= & 64 \theta_{1} \sum_{n=1}^{\infty} \sum_{m=1}^{\infty}\left(\frac{1}{\alpha_{n}{ }^{2} L \ell}+\frac{1}{\gamma_{m}{ }^{2} L \ell}\right) \frac{1}{\delta_{n m}{ }^{2} L \ell} \\
& \times\left\{\left[\frac{\delta_{n m}{ }^{2} h^{2}}{\alpha_{n}{ }^{2} h^{2}+\gamma_{m}{ }^{2} h^{2}}-1\right] \frac{2}{\delta_{n m} h} \frac{\sinh \left(\delta_{n m} \frac{h}{2}\right)}{\cosh \left(\delta_{n m} \frac{h}{2}\right)}+1\right\} .
\end{aligned}
$$

Introducing this last result back into Eq. (A33) provides the expression of $\theta_{1}$ given by

$$
\theta_{1}=\frac{R T}{M\left(V_{1}-V\right)} \frac{\Phi_{1}}{p} \frac{1}{1+64 \frac{\varepsilon V}{V_{1}-V} \sum_{n=1}^{\infty} \sum_{m=1}^{\infty} F_{n m}},
$$

in which $F_{n m}$ is given by

$$
\begin{aligned}
F_{n m}= & \left(\frac{1}{\alpha_{n}^{2} L \ell}+\frac{1}{\gamma_{m}^{2} L \ell}\right) \frac{1}{\delta_{n m}^{2} L \ell}\left\{\left[\frac{\delta_{n m}{ }^{2} h^{2}}{\alpha_{n}{ }^{2} h^{2}+\gamma_{m}{ }^{2} h^{2}}-1\right]\right. \\
& \left.\times \frac{2}{\delta_{n m} h} \frac{\sinh \left(\delta_{n m} \frac{h}{2}\right)}{\cosh \left(\delta_{n m} \frac{h}{2}\right)}+1\right\} .
\end{aligned}
$$

The solution for $P_{1}(t)$ is finally obtained from an inverse Laplace transform of Eq. (A36).

\section{APPENDIX B: SOLUTION FOR A CYLINDRICAL SAMPLE}

The objective of this appendix is to provide the solution of the initial boundary value problem governing the pressure evolution in the sample $(P)$ and in tank $1\left[P_{1}(t)\right]$ for a cylindrical sample of circular cross section with all its faces left open. Using the notations $\bar{P}=P-P_{i}$ and $\bar{P}_{1}=P_{1}-P_{i}$ and the definition of $a$ given in Eq. (11), the system of equations can be written as

$$
\begin{aligned}
& \frac{\partial^{2} \bar{P}}{\partial x^{2}}+\frac{1}{r} \frac{\partial \bar{P}}{\partial r}+\frac{\partial^{2} \bar{P}}{\partial r^{2}}=\frac{1}{a} \frac{\partial \bar{P}}{\partial t}, \\
& \text { B.C.1 } \frac{\partial \bar{P}}{\partial x}=0 \quad \text { at } x=0 \text {, } \\
& \text { B.C.2 } \bar{P}=P_{1}(t)-P_{i}=\bar{P}_{1}(t) \quad \text { at } x=\frac{L}{2} \text { and } r=r_{1} \text {, } \\
& \text { I.C. } \\
& \bar{P}=\bar{P}_{1}=0 \quad \text { at } t=0 .
\end{aligned}
$$

The constraint imposing that $\bar{P}$ remains finite at $r=0$ must be added to these equations. The complete statement of the problem requires the expression of the mass balance of gas in the system, which takes the following form:

$$
\frac{M}{R T}\left(V_{1}-V\right) \frac{d \bar{P}_{1}}{d t}=-\varepsilon V \frac{M}{R T} \frac{d \bar{P}_{m}}{d t}+\dot{q}_{m 0} .
$$

The solution is carried out by first applying a Laplace transform to the above equations. Using the nomenclature $\theta=\mathcal{L}(\bar{P})$ $=\int_{0}^{\infty} \bar{P} \exp (-p t) d t$, this yields

$$
\begin{gathered}
\frac{\partial^{2} \theta}{\partial x^{2}}+\frac{1}{r} \frac{\partial \theta}{\partial r}+\frac{\partial^{2} \theta}{\partial r^{2}}=\frac{p}{a} \theta, \\
\frac{\partial \theta}{\partial x}=0 \quad \text { at } x=0, \\
\theta=\theta_{1} \quad \text { at } x=\frac{L}{2} \text { and } r=r_{1}, \\
\frac{M}{R T}\left(V_{1}-V\right) p \theta_{1}+\varepsilon V \frac{M}{R T} p \theta_{m}=\Phi_{1},
\end{gathered}
$$

while $\theta$ remains finite at $r=0$.

At this point, a cosine Fourier transform, defined as $\bar{\theta}$ $=\int_{0}^{\frac{L}{2}} \theta \cos \left(\alpha_{n} x\right) d x$ with the eigenvalues $\alpha_{n}$, given by $\alpha_{n}=\frac{(2 n-1) \pi}{L}$, is applied to Eq. (B6), yielding

$$
\int_{0}^{\frac{L}{2}} \frac{\partial^{2} \theta}{\partial x^{2}} \cos \left(\alpha_{n} x\right) d x+\frac{1}{r} \frac{\partial \bar{\theta}}{\partial r}+\frac{\partial^{2} \bar{\theta}}{\partial r^{2}}=\frac{p}{a} \bar{\theta} .
$$


Carrying out the integration of the first term on the left-hand side of the above equation leads to

$$
\frac{1}{r} \frac{\partial \bar{\theta}}{\partial r}+\frac{\partial^{2} \bar{\theta}}{\partial r^{2}}-\delta_{n}{ }^{2} \bar{\theta}+\alpha_{n} \theta_{1} \sin \left(\alpha_{n} \frac{L}{2}\right)=0
$$

with

$$
\delta_{n}^{2}=\alpha_{n}^{2}+\frac{p}{a} .
$$

The solution of Eq. (B11) is given by

$$
\bar{\theta}=A I_{0}\left(\delta_{n} r\right)+R,
$$

where $I_{0}$ is the zeroth order Bessel function of the first kind and $R$ is given by

$$
R=\frac{\alpha_{n} \theta_{1} \sin \left(\alpha_{n} \frac{L}{2}\right)}{\delta_{n}^{2}} .
$$

The Fourier transform applied to the boundary condition at $r=r_{1}$ [Eq. (B8)] yields

$$
\bar{\theta}=\theta_{1} \frac{1}{\alpha_{n}} \sin \left(\alpha_{n} \frac{L}{2}\right) \quad \text { at } r=r_{1}
$$

This allows one to determine $\mathrm{A}$, which is given by

$$
A=\frac{\theta_{1}}{I_{0}\left(\delta_{n} r_{1}\right)}\left(\frac{1}{\alpha_{n}}-\frac{\alpha_{n}}{\delta_{n}^{2}}\right) \sin \left(\alpha_{n} \frac{L}{2}\right)
$$

Thus, the solution for $\bar{\theta}$ is

$$
\bar{\theta}=\theta_{1}\left(\frac{1}{\alpha_{n}}-\frac{\alpha_{n}}{\delta_{n}{ }^{2}}\right) \frac{I_{0}\left(\delta_{n} r\right)}{I_{0}\left(\delta_{n} r_{1}\right)} \sin \left(\alpha_{n} \frac{L}{2}\right)+\frac{\alpha_{n} \theta_{1} \sin \left(\alpha_{n} \frac{L}{2}\right)}{\delta_{n}{ }^{2}} .
$$

Using the definition of the Fourier transform, the solution for $\theta$ is given by

$$
\theta=4 \sum_{n=1}^{\infty} \frac{\bar{\theta} \cos \left(\alpha_{n} x\right)}{L}
$$

This solution can now be used to express $\theta_{m}$ as

$$
\theta_{m}=\frac{4}{r_{1}^{2} L} \int_{0}^{\frac{L}{2}} \int_{0}^{r_{1}} \theta r d r d x
$$

Carrying out the two integrations leads to

$$
\begin{aligned}
\theta_{m}= & \sum_{n=1}^{\infty} \frac{4}{\alpha_{n} L} \theta_{1} \sin \left(\alpha_{n} \frac{L}{2}\right)\left[\left(\frac{1}{\alpha_{n}}-\frac{\alpha_{n}}{\delta_{n}{ }^{2}}\right) \sin \left(\alpha_{n} \frac{L}{2}\right) \frac{r_{1}}{\delta_{n}} \frac{I_{1}\left(\delta_{n} r_{1}\right)}{I_{0}\left(\delta_{n} r_{1}\right)}\right. \\
& \left.+\frac{\alpha_{n}}{\delta_{n}{ }^{2}} \sin \left(\alpha_{n} \frac{L}{2}\right) \frac{r_{1}{ }^{2}}{2}\right]
\end{aligned}
$$

with $I_{1}$ the first order Bessel function of the first kind and, according to the definition of the eigenvalues, $\sin ^{2}\left(\alpha_{n} \frac{L}{2}\right)=1$. This allows one to rewrite $\theta_{m}$ as

$$
\theta_{m}=16 \theta_{1} \sum_{n=1}^{\infty} \frac{1}{\delta_{n} L}\left[\frac{L}{r_{1}}\left(\frac{1}{\alpha_{n}^{2} L^{2}}-\frac{1}{\delta_{n}^{2} L^{2}}\right) \frac{I_{1}\left(\delta_{n} r_{1}\right)}{I_{0}\left(\delta_{n} r_{1}\right)}+\frac{1}{2} \frac{1}{\delta_{n} L}\right] .
$$

Introducing this result back into Eq. (B9) yields

$$
\begin{aligned}
\theta_{1}\left[\frac{M}{R T}\left(V_{1}-V\right) p+8 \varepsilon V \frac{M}{R T} p \sum_{n=1}^{\infty} \frac{1}{\delta_{n}^{2} L^{2}}\right. \\
\left.\times\left[2 \frac{L}{r_{1}}\left(\frac{\delta_{n} L}{\alpha_{n}^{2} L^{2}}-\frac{1}{\delta_{n} L}\right) \frac{I_{1}\left(\delta_{n} r_{1}\right)}{I_{0}\left(\delta_{n} r_{1}\right)}+1\right]\right]=\Phi_{1},
\end{aligned}
$$

from which the solution for $\theta_{1}$ can be finally extracted to give

$$
\begin{aligned}
\theta_{1}= & \frac{\Phi_{1}(p)}{p} \frac{R T}{M\left(V_{1}-V\right)} \\
& \times \frac{1}{1+8 \frac{\varepsilon V}{V_{1}-V} \sum_{n=1}^{\infty} \frac{1}{\delta_{n}{ }^{2} L^{2}}\left[2 \frac{L}{r_{1}}\left(\frac{\delta_{n} L}{\alpha_{n}{ }^{2} L^{2}}-\frac{1}{\delta_{n} L}\right) \frac{I_{1}\left(\delta_{n} r_{1}\right)}{I_{0}\left(\delta_{n} r_{1}\right)}+1\right]} .
\end{aligned}
$$

\section{DATA AVAILABILITY}

The data that support the findings of this study are available from the corresponding author upon reasonable request.

\section{REFERENCES}

${ }^{1}$ R. Sander, Z. Pan, and L. D. Connell, "Laboratory measurement of low permeability unconventional gas reservoir rocks: A review of experimental methods," J. Nat. Gas Sci. Eng. 37, 248-279 (2017).

${ }^{2}$ G. Gaus, A. Amann-Hildenbrand, B. M. Krooss, and R. Fink, "Gas permeability tests on core plugs from unconventional reservoir rocks under controlled stress: A comparison of different transient methods," J. Nat. Gas Sci. Eng. 65, 224-236 (2019).

${ }^{3}$ C. Jones and P. Meredith, "An experimental study of elastic propagation anisotropy in an illitic shale," in Paper Presented at the SPE/IRSM Eurock, Trondheim, Norway, 8-10 July 1998.

${ }^{4}$ P. F. Boulin, P. Bretonnier, N. Gland, and J. M. Lombard, "Contribution of the steady state method to water permeability measurement in very low permeability porous media," Oil Gas Sci. Technol. 67(3), 387-401 (2012).

${ }^{5}$ X. Cui, A. M. M. Bustin, and R. M. Bustin, "Measurements of gas permeability and diffusivity of tight reservoir rocks: Different approaches and their applications," Geofluids $\mathbf{9}, 208-223$ (2009).

${ }^{6}$ W. F. Brace, J. B. Walsh, and W. T. Frangos, "Permeability of granite under high pressure," J. Geophys. Res. 73(6), 2225-2236, https://doi.org/10.1029/jb073i006p02225 (1968).

${ }^{7}$ A. I. Dicker and R. M. Smits, "A practical approach for determining permeability from laboratory pressure-pulse decay measurements," in Paper Presented at the International Meeting on Petroleum Engineering, Tianjin, China, 1-4 November 1988.

${ }^{8}$ S. C. Jones, "A rapid accurate unsteady-state permeameter Klinkenberg permeameter,” Soc. Pet. Eng. J. 12(5), 383-397 (1972).

${ }^{9}$ S. C. Jones, "A technique for faster pulse decay permeability measurements in tight rocks,” SPE Form. Eval. 12, 19-25 (1997).

${ }^{10}$ R. L. Kranz, J. S. Saltzman, and J. D. Blacic, "Hydraulic diffusivity measurements on laboratory rock samples using an oscillating pore pressure method," Int. J. Rock Mech. Min. Sci. Geomech. Abstr. 27(5), 345-352 (1990).

${ }^{11}$ G. J. Fischer, "Chapter 8-The determination of permeability and storage capacity: Pore pressure oscillation method," Fault Mechanics and Transport Properties of Rocks (Academic Press, 1992).

${ }^{12}$ A. K. Hasanov, B. Dugan, and M. L. Batzle, "Numerical simulation of oscillating pore pressure experiments and inversion for permeability," Water Resour. Res. 56(6), e2019WR025681, https://doi.org/10.1029/2019wr025681 (2020).

${ }^{13}$ D. Lasseux and Y. Jannot, "Measurement of parameters linked to the flow of fluids in a porous material," Patent WO2011089367 CNRS-TOTAL (July 28, 2011), see https://worldwide.espacenet.com/publicationDetails/biblio?CC=WO \& NR= $2011089367 \& \mathrm{KC}=$ \& $\mathrm{FT}=\mathrm{E}$ \& locale=en_EP\#.

${ }^{14}$ D. Lasseux, Y. Jannot, S. Profice, M. Mallet, and G. Hamon, “The 'step decay': A new transient method for the simultaneous determination of intrinsic permeability, Klinkenberg coefficient and porosity on very tight rocks," in Paper Presented at 
the International Symposium of the Society of Core Analysts, Aberdeen, Scotland, 27-30 August 2012.

${ }^{15}$ D. Lasseux, F. J. Valdés Parada, J. A. O. Tapia, and B. Goyeau, "A macroscopic model for slightly compressible gas slip flow in homogeneous porous media," Phys. Fluids 26(5), 053102 (2014).

${ }^{16}$ D. Lasseux, F. J. Valdés Parada, and M. L. Porter, "An improved macroscale model for gas slip flow in porous media," J. Fluid Mech. 805, 118-146 (2016).

${ }^{17} \mathrm{~L}$. J. Klinkenberg, "The permeability of porous media to liquids and gases, drilling and production practice," in Drilling and Production Practice (American Petroleum Institute, 1941), pp. 200-213.

${ }^{18}$ D. L. Luffel and F. K. Guidry, Core analysis results, comprehensive study wells, Devonian shales, GRI Topical Report No. 129, Gas Research Institute, Houston, Des Plaines, IL, 1989.

${ }^{19}$ D. L. Luffel and F. K. Guidry, "New core analysis methods for measuring reservoir rock properties of Devonian shale," J. Pet. Technol. 44(11), 1184-1190 (1992).

${ }^{20}$ D. L. Luffel, C. W. Hopkins, and P. D. Schettler, Jr., "Matrix permeability measurement of gas productive shales," in Paper Presented at the 68th SPE Annual Technical Conference and Exhibition, Houston, Texas, 3-6 October 1993.

${ }^{21} \mathrm{~K}$. Guidry, D. Luffel, and J. Curtis, Development of laboratory and petrophysical techniques for evaluating shale reservoirs. Final Report No. GRI-95/0496: 304, Gas Research Institute, Des Plaines, IL, 1995.

${ }^{22} \mathrm{~A}$. Cui and R. Brezovski, "Laboratory permeability and diffusivity measurements of unconventional reservoirs: Useless or full of information? A montney example from the western Canadian sedimentary basin," in Paper Presented at the SPE Asia Pacific Unconventional Resources Conference and Exhibition, Brisbane, Australia, 11-13 November 2013.

${ }^{23}$ S. Profice, D. Lasseux, Y. Jannot, N. Jebara, and G. Hamon, "Permeability, porosity and Klinkenberg coefficient determination on crushed porous media," Petrophysics 53(6), 430-438 (2012).

${ }^{24}$ Y. Jannot, D. Lasseux, G. Vizé, and G. Hamon, "A detailed analysis of permeability and Klinkenberg coefficient estimation from unsteady state pulse decay or draw down experiments," in Society of Core Analysis Symposium, Calgary, 9-14 September 2007.

${ }^{25}$ Y. Jannot and D. Lasseux, "A new quasi-steady method to measure gas permeability of weakly permeable porous media," Rev. Sci. Instrum. 83, 015113 (2012).

${ }^{26}$ Y. Jannot and C. Moyne, Transferts Thermiques: Cours Et 55 Exercices Corrigés (Edilivre, 2014).

${ }^{27}$ Y. Jannot and A. Degiovanni, Thermal Properties Measurement of Materials (John Wiley \& Sons, 2018).

${ }^{28}$ F. R. De Hoog, "An improved method for numerical inversion of Laplace transforms," J. Soc. Ind. Appl. Math. 3(3), 357-366 (1982).

${ }^{29} \mathrm{H}$. Bal, Y. Jannot, N. Quenette, A. Chenu, and S. Gaye, "Water content dependence of the porosity, density and thermal capacity of laterite based bricks with millet waste additive," Constr. Build. Mater. 31, 144-150 (2012).

${ }^{30} \mathrm{D}$. W. Marquart, "An algorithm for least squares estimation of non-linear parameters," J. Soc. Ind. Appl. Math. 11, 431-441 (1963). 